\title{
Experimental Search for Pentaquarks
}

\author{
Kenneth H. Hicks \\ Department of Physics and Astronomy, Ohio University, Athens, OH 45701, USA
}

November 11, 2018

\begin{abstract}
The experimental evidence for pentaquarks, both old and new, is discussed. Constraints due to $\mathrm{K}^{+} \mathrm{N}$ scattering data from previous decades is first reviewed, followed by experiments with positive evidence and those with null results. Finally, the problem of the narrow width of the $\Theta^{+}$pentaquark is discussed, along with theoretical implications.
\end{abstract}

\section{Introduction}

There is a well-known saying that extraordinary claims require extraordinary proof. In the world of hadron spectroscopy, the discovery of a new class of hadrons (that cannot be described in terms of the standard classes: 3-quark baryons or quark-antiquark mesons) could be called an extraordinary claim. In fact, ten experiments claim positive evidence for a new hadron called the $\Theta^{+}$(see Table 1), which has the quantum numbers of a pentaquark made from two up quarks, two down quarks and one strange antiquark (structure $u u d d \bar{s}$ ). However, no single experiment meets the criteria of extraordinary proof expected for such a claim. In addition, there are a number of high-energy experiments that did not observe the $\Theta^{+}$(see Table 2) when naive estimates suggest it should have been seen. Hence, there is reasonable doubt that the $\Theta^{+}$ exists.

There have also been searches for other pentaquarks with different quarkflavor composition. Evidence for the $\Xi^{--}$pentaquark $(d d s s \bar{u})$ has been seen by one experiment [22, but has not been confirmed in other experiments [23, 24]. Evidence for the $\Theta_{c}$, where the $\bar{c}$ quark replaces the $\bar{s}$ in the $\Theta^{+}$, has been published [25], but again there is no confirmation [26]. Little more will be said about these observations, since confirmation is first desired.

The purpose of this review is to provide the reader with the experimental facts up to the present time (i.e. the end of 2004). However, the field is developing rapidly [27, and this review is likely to be outdated before it is published.

On a broader scale, the search for the pentaquark is an example of science at work, where initial evidence is peer-reviewed, published and then checked in all possible ways. Some claims survive and some fail. The fate of the $\Theta^{+}$ pentaquark is yet to be determined. 
Table 1: Published experiments with evidence for the $\Theta^{+}$baryon.

\begin{tabular}{|c|l|c|c|c|c|}
\hline Reference & Group & Reaction & $\begin{array}{c}\text { Mass } \\
(\mathrm{MeV})\end{array}$ & $\begin{array}{c}\text { Width } \\
(\mathrm{MeV})\end{array}$ & $\sigma^{\prime}$ s* $^{*}$ \\
\hline$[1]$ & LEPS & $\gamma C \rightarrow K^{+} K^{-} X$ & $1540 \pm 10$ & $<25$ & 4.6 \\
{$[2]$} & DIANA & $K^{+} X e \rightarrow K^{0} p X$ & $1539 \pm 2$ & $<9$ & 4.4 \\
{$[3]$} & CLAS & $\gamma d \rightarrow K^{+} K^{-} p(n)$ & $1542 \pm 5$ & $<21$ & $5.2 \pm 0.6^{\dagger}$ \\
{$[4]$} & SAPHIR & $\gamma d \rightarrow K^{+} K^{0}(n)$ & $1540 \pm 6$ & $<25$ & 4.8 \\
{$[5]$} & ITEP & $\nu A \rightarrow K^{0} p X$ & $1533 \pm 5$ & $<20$ & 6.7 \\
{$[6]$} & CLAS & $\gamma p \rightarrow \pi^{+} K^{+} K^{-}(n)$ & $1555 \pm 10$ & $<26$ & 7.8 \\
{$[7]$} & HERMES & $e^{+} d \rightarrow K^{0} p X$ & $1526 \pm 3$ & $13 \pm 9$ & $\sim 5$ \\
{$[8]$} & ZEUS & $e^{+} p \rightarrow e^{+} K^{0} p X$ & $1522 \pm 3$ & $8 \pm 4$ & $\sim 5$ \\
{$[9]$} & COSY-TOF & $p p \rightarrow K^{0} p \Sigma^{+}$ & $1530 \pm 5$ & $<18$ & $4-6$ \\
{$[10]$} & SVD & $p A \rightarrow K^{0} p X$ & $1526 \pm 5$ & $<24$ & 5.6 \\
\hline
\end{tabular}

* Gaussian fluctuation of the background, as $N_{\text {peak }} / \sqrt{N_{B G}}$. This "naive" significance may underestimate the real probability of a fluctuation by about 1-2 $\sigma$.

$\dagger$ Further analysis of the CLAS deuterium data suggest that the significance of the observed peak may not be as large as indicated.

Table 2: Published experiments with non-observation of the $\Theta^{+}$baryon.

\begin{tabular}{|c|l|c|c|c|}
\hline Reference & Group & Reaction & Limit & Sensitivity? \\
\hline$[11]$ & BES & $e^{+} e^{-} \rightarrow J / \Psi \rightarrow \Theta \Theta$ & $<1.1 \times 10^{-5}$ B.R. & No [68] \\
\hline$[12]$ & BaBar & $e^{+} e^{-} \rightarrow \Upsilon(4 S) \rightarrow p K^{0} X$ & $<1.0 \times 10^{-4}$ B.R. & Maybe \\
{$[13]$} & Belle & $e^{+} e^{-} \rightarrow B^{0} \bar{B}^{0} \rightarrow p \bar{p} K^{0} X$ & $<2.3 \times 10^{-7}$ B.R. & No \\
{$[1]$} & LEP & $e^{+} e^{-} \rightarrow Z \rightarrow p K^{0} X$ & $<6.2 \times 10^{-4}$ B.R. & No? \\
\hline$[15]$ & HERA-B & $p A \rightarrow K^{0} p X$ & $<0.02 \times \Lambda^{*}$ & No? \\
{$[16]$} & SPHINX & $p C \rightarrow K^{0} \Theta^{+} X$ & $<0.1 \times \Lambda^{*}$ & Maybe \\
{$[17]$} & HyperCP & $p C u \rightarrow K^{0} p X$ & $<0.3 \% K^{0} p$ & No? \\
{$[18]$} & CDF & $p \bar{p} \rightarrow K^{0} p X$ & $<0.03 \times \Lambda^{*}$ & No? \\
{$[19]$} & FOCUS & $\gamma B e O \rightarrow K^{0} p X$ & $<0.02 \times \Sigma^{*}$ & Maybe \\
{$[20]$} & Belle & $\pi+S i \rightarrow K^{0} p X$ & $<0.02 \times \Lambda^{*}$ & Yes? \\
{$[21]$} & PHENIX & $A u+A u \rightarrow K^{-} \bar{n} X$ & (not given) & Unknown \\
\hline
\end{tabular}




\subsection{Preliminaries}

A simplified definition of a pentaquark is a particle with a valence structure of four quarks and one antiquark. Quantum chromodynamics (QCD) does not forbid multiquark particles, as long as they are colorless. Because pentaquarks can decay ("fall apart" mode) into a three-quark baryon and a quark-antiquark meson, pentaquarks were expected [28, 29] to have wide widths. This would be difficult to observe experimentally. However, some theorists [30, 31] suggested that particular quark structures might exist with a narrow width. This led to renewed interest in experimental searches for pentaquarks.

Why is it important to know whether pentaquarks (with narrow widths) exist? If they do exist, then we have a new multiquark system which can be used to test the theory of quantum chromodynamics (QCD) in the nonperturbative regime. Until now, most of the effort for calculations of nonperturbative QCD have focused on baryons and mesons. Several question come to mind. How tightly bound are multiquark systems? How much overlap does the wavefunction of a multiquark particle have with the final decay state? These questions show that pentaquarks could provide a new testbed for QCD. In particular, lattice QCD has recently produced (in the quenched approximation) a spectrum of baryon resonances [32, and similar studies of pentaquarks on the lattice are underway (see the review by Sasaki 33]).

If pentaquarks with a narrow width exist, then we will learn more about the effective forces between quarks and whether lattice QCD calculations can reproduce the data. So it is important to do experimental searches for pentaquarks whether or not you believe in any particular theory that predicts a given multiquark state.

It is natural to split the effort of a review of the renewed interest in pentaquarks into experimental an theoretical aspects. The latter is reviewed in a separate article that follows in the same volume [34, where it is shown that there are still many theoretical questions that surround the possibility of a narrow pentaquark resonance. But experiments must first show that (narrow) pentaquarks really exist. Experiment is the focus of the current article.

Since there were many experiments done in previous decades, new searches should have advantages not previously available. For example, new photoproduction facilities are now able to gather data for multiparticle final states at least an order of magnitude better than before [35. This opens the door to new precision measurements and detection of weakly-produced states not seen before. With theoretical guidance, it is again reasonable to look at new data and search for evidence of narrow pentaquark states.

\section{Kaon Nucleon Scattering Data}

The $\Theta^{+}$resonance (if it exists) has the same quarks as a the combination of a $K^{+}$meson together with a neutron. Another way to divide the quarks gives the combination of a $K^{0}$ and a proton. These two combinations are expected to be the primary decay branches of the $\Theta^{+}$and will have equal amplitudes by isospin symmetry. Reversing the process, the $\Theta^{+}$can be made by putting a $K^{+}$ 


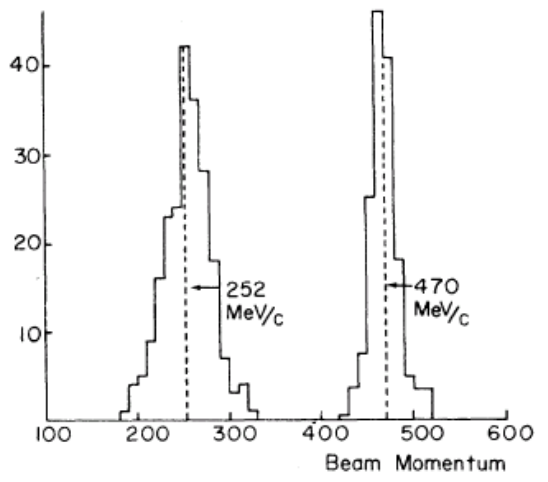

Figure 1: The $K^{+}$beam momentum distributions for Ref. 38. Note that $470 \mathrm{MeV} / \mathrm{c}$ corresponds to a center-of-mass energy near $1550 \mathrm{MeV}$, a bit above the average mass or the $\Theta^{+}$in Table 1 .

beam onto a neutron (using deuterium or another nuclear target) or a $K^{0}$ beam onto a proton. A $K^{0}$ beam is difficult to produce, but there is some data (see the references in [36]) albeit with large uncertainties. A more promising avenue is to examine $K^{+} d$ scattering. Next we review the $K^{+} N$ scattering data, which is taken mostly from bubble-chamber experiments in the 1960's and 1970's.

\subsection{Partial wave analysis}

Let us begin with one partial wave analysis (PWA) of the $K^{+} N$ scattering done in 1992 [37. In this paper [37] there is a compendium of the $K^{+} N$ scattering database. Only some of the data has a deuteron target and most of these are at beam energies above about $200 \mathrm{MeV}$. (The average mass of the $\Theta^{+}$shown in Table 1 is about $1535 \mathrm{MeV}$, which is about $100 \mathrm{MeV}$ above the $K^{+} N$ threshold.) Higher energy $K^{+}$beams result in less $K^{+}$decays on the way to the target, and hence typically have higher intensity and higher purity. Most lower-energy $K^{+} N$ measurements were done by starting with a higher-energy beam, and then decelerating the beam using energy loss in the target or designed energy degraders. The resulting beam energy can have significant momentum spread, as in Fig. 1] where two of the four momenta measured in Ref. 38] are shown.

It turns out that the $K^{+} d$ scattering database has sparse coverage in the region of the $\Theta^{+}$mass. An additional complication is that there is no neutron target, and so the isospin $I=0$ amplitude must be extracted from deuterium data, containing a mixture of $I=0$ and $I=1$ amplitudes. This can be done only after correcting the data for the Fermi motion of the target nucleons (and the momentum spread of the $K^{+}$beam). These unfolding procedures are straight-forward if one has sufficiently precise measurements at small steps in the beam energy. An example of the Fermi motion correction is given in Bowen et al. 39] where structure in the energy-dependence of the total cross section is evident only after this procedure. Furthermore, the database is "noisy" with 


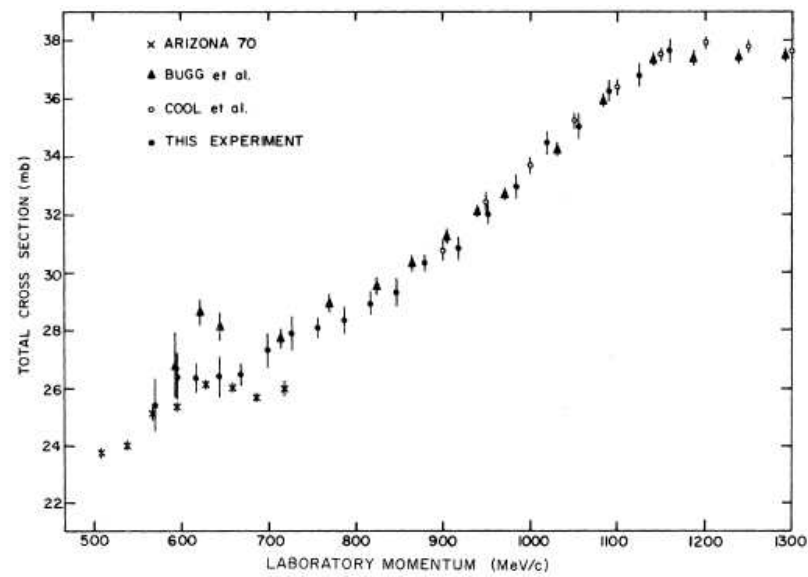

Figure 2: Total cross sections for $K^{+} d$ scattering taken from Ref. [40]. In the region below $700 \mathrm{MeV} / \mathrm{c}$, different measurements disagree significantly.

significant disagreement in the regions of overlap from independent measurements. This is shown in Fig. [2 where several measurements of the $K^{+} d$ total cross section disagree below a beam momentum of $700 \mathrm{MeV} / \mathrm{c}$. This is not the only case, as the integrated cross sections of Glasser et al. 38 at 470 $\mathrm{MeV} / \mathrm{c}$ (when coherent, breakup and charge-exchange are added together) is more than $10 \%$ below the total cross sections of Bowen et al. in the same momentum range, a difference which is much larger than the systematic errors of either experiment. Hence, one must be careful when drawing any conclusions from this database.

With these caveats in mind, we return to the partial wave analysis. In the original UCLA/Berkeley papers from the 1960's [41] they conclude that the $I=0$ s-wave phase shifts (fit to their $K^{+} d$ data from 312 to $812 \mathrm{MeV} / \mathrm{c}$ ) are attractive, indicating possible resonance structure, and for the $I=0 \mathrm{p}$-waves there are two solutions. The question of which solution to use was later resolved using polarization data 42 from Brookhaven, giving substantial attraction in the $P_{1 / 2}$ partial wave. Moving to 1977, Glasser et al. 38. found again attraction in the $P_{1 / 2}$ partial wave, but now the s-wave solution was repulsive at lower beam momenta (342 and $470 \mathrm{MeV} / \mathrm{c}$ ), turning attractive at $587 \mathrm{MeV} / \mathrm{c}$. Now using the full database as of 1993, Hyslop et al. [37] found that the s-wave is repulsive for all beam momenta, and attraction in the $P_{1 / 2}$ and also the $P_{3 / 2}$ partial waves (the latter was repulsive for Ref. 41] and nearly zero for Ref. [38]). A more recent paper by Barnes and Swanson [43. agree with Hyslop, giving a repulsive s-wave, but did not report the p-wave solutions.

What can we conclude from this detour into history? That the database is indeed noisy, and one must be careful to look at the individual measurements. The "average" phase shifts that fit the whole database may not fit each data set. As an example, the $K^{+} d \rightarrow K^{+} n(p)$ data of Damerell et al. 44] are shown in Fig. 3 for the four lowest beam momenta. At the lowest momenta, the data are significantly underestimated by the overall PWA solution. Better measurements for $K^{+} N$ scattering are needed, especially at low energy [43]. It is possible that some data sets are erroneous, but we cannot know without 


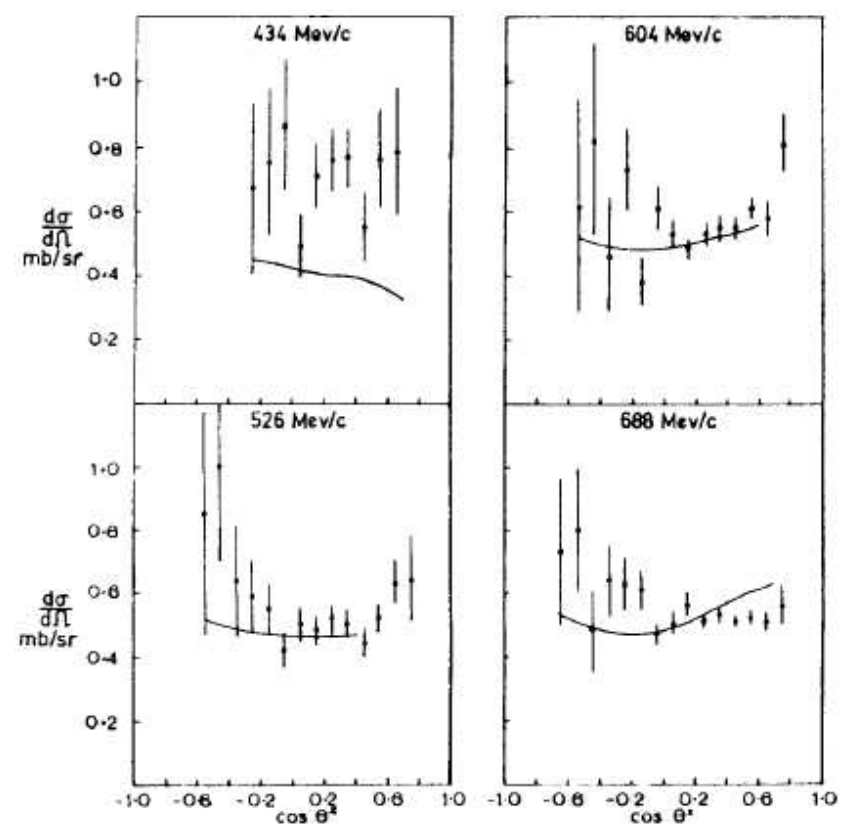

Figure 3: Differential cross sections for the $K^{+} d \rightarrow K^{+} n(p)$ reaction from Ref. [44. The curves are PWA phase shift solutions. Note that the $434 \mathrm{MeV} / \mathrm{c}$ data are underestimated by a factor of about 2 .

better data.

\subsection{Connection to the $\Theta^{+}$width}

The main point to consider here is whether the $K^{+} N$ database is consistent with the existence of a narrow $\Theta^{+}$resonance. Shortly after the first $\Theta^{+}$papers [1]- [4] were published, some comparisons with the $K^{+} N$ data were done. Nussinov 45. was one of the first, and based on the general expression for the $K^{+} n$ total cross section evaluated on-resonance (with the phase shift at $90^{\circ}$ ) and the momentum needed to reach the $\Theta^{+}$mass, he finds a $37 \mathrm{mb}$ value. If the $\Theta^{+}$ is narrow, it could escape detection if there is a gap in the database at the resonant energy, but the deuteron's Fermi motion will spread it out so that it should be noticeable. Using these estimates and a cursory examination of the database, Nussinov concludes that the width of the $\Theta^{+}$must be less than 6 $\mathrm{MeV}$. Other estimates of the width followed, using similar but perhaps more careful approaches [46, 47, 48, 49, 50] and all of these agree that the $\Theta^{+}$width must be less than a few $\mathrm{MeV}$ to be consistent with the $K^{+} N$ total cross section.

One comparison to the KN database by Gibbs [50] is particularly interesting. This paper is based on a weak scattering approximation and the resulting calculation is compared with the total cross section data of Ref. [39], as shown in Fig. 4 The dotted curve is for non-resonant background, and the other three curves correspond to $\Theta^{+}$widths of $0.6,0.9$ and $1.2 \mathrm{MeV}$ for a positive parity resonance of mass about $1.56 \mathrm{GeV}$. This resonance mass was obtained as the best fit to the data. Of course, the uncertainties in the data allow rea- 


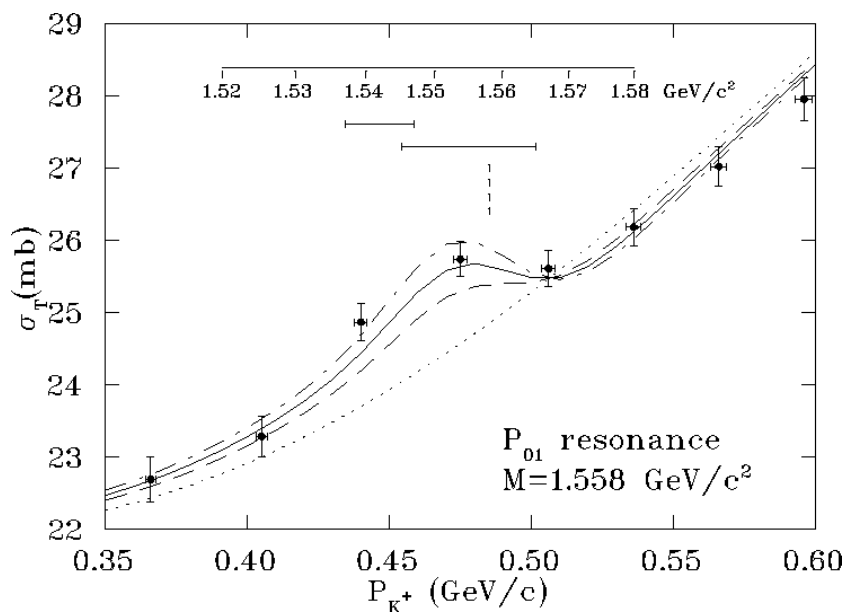

Figure 4: Fits to the total cross sections of Ref. [39] by W. Gibbs [50] using a weakscattering approximation, assuming a positive-parity resonance at the mass and width shown.

sonable $\chi^{2}$ values down to a mass of about $1.545 \mathrm{GeV}$. Assuming a negative parity resonance gives lower mass, shown by the second horizontal bar near the top of the figure. In all cases, the width of the $\Theta^{+}$must be unusually small, on the order of $1 \mathrm{MeV}$. If the $\Theta^{+}$exists with such a small width, then theoretical models of the quark structure of the $\Theta^{+}$become highly constrained (see section $5)$.

\subsection{Possible resonant structure}

There is stronger evidence at higher masses that there is resonant structure in the $K^{+} N$ total cross sections. In Fig. 5 the isoscalar cross section, $\sigma_{0}$ has been extracted from the $K^{+} d$ total cross section of Cool et al. [51 and Bugg et al [52. Here, there are two resonance structures, one at a mass of about 1710 $\mathrm{MeV}$ and another at about $1860 \mathrm{MeV}$. The $1860 \mathrm{MeV}$ resonance was also found in an early photoproduction experiment [53] with a width of about $150 \mathrm{MeV}$. Higher-mass resonances were also found in the PWA of Hyslop et al [37] but have not received much attention. Some experimental effort devoted to looking for these resonances using modern photoproduction facilities would make sense.

One final comment about the $\mathrm{KN}$ data is from the paper by Berthon et al. 54 for the reaction $K^{+} p \rightarrow p K_{s}^{0} \pi^{+}$. This bubble chamber experiment was done at several incident kaon energies, with the highest momentum shown in Fig. 6] This figure shows several combinations of invariant mass of final state particles, for $M\left(p \pi^{+}\right), M\left(K^{0} \pi^{+}\right)$and $M\left(p K^{0}\right)$. The first shows a broad peak near the $\Delta(1232)$ mass, the second shows a clear peak at the mass of the $K^{*}(892)$ vector meson, and the third has a small shoulder at $M^{2}=2.35 \mathrm{GeV}^{2}$ (or $M=1.54 \mathrm{GeV}$ ). However, further examination of the Dalitz plot for this reaction does not show any resonance structure, and so it is possible that this small shoulder in the mass distribution is just a statistical fluctuation. Better data for this reaction is desired. An experiment at KEK [55] for the $H\left(K^{+}, \pi^{+}\right)$ 


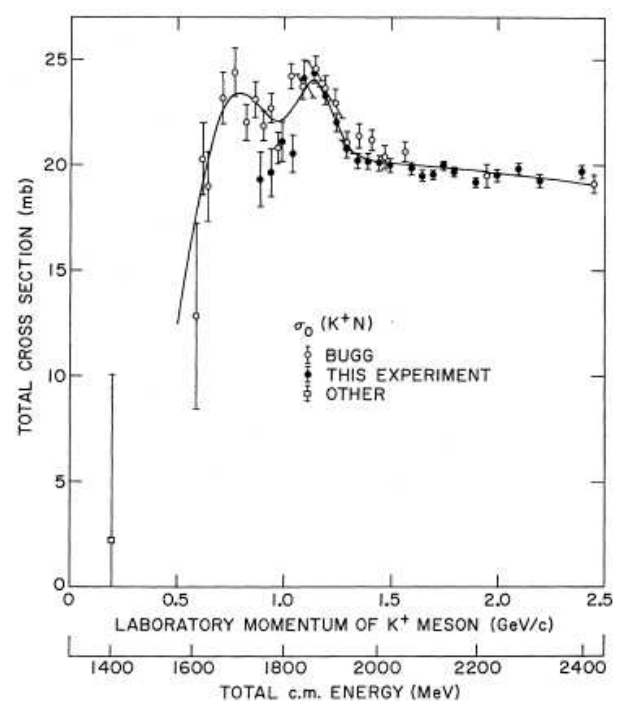

Figure 5: Data of Refs. 51] and [52 for the isoscalar total cross section extracted from $K^{+} d$ scattering data. The resonant-like structures at masses of about 1710 and $1850 \mathrm{MeV}$ are also identified in photoproduction [53].

reaction has been approved and is scheduled to run in May 2005.

\section{Positive evidence for the $\Theta^{+}$}

There have been many articles describing the discovery of the pentaquark known as the $\Theta^{+}$at the SPring- 8 facility in Japan. For more details about the initial discovery and two confirming experiments, please see Ref. [56].

A montage of the data from the experiments listed in Table 1 is shown in Fig. 7 Here, the data are plotted with error bars and without fits to guide the eye. When plotted this way, it is clear that better statistics are needed, since no single result shows a really convincing peak. On the other hand, taken as a whole, there seems to be something in the data at $1535 \mathrm{MeV}$, independent of the probe or detector. Whether this might be due to coincidental statistical fluctuations, or some effect from the event selection in the analysis, is a serious question. If we assume the experiments were analyzed properly then it seems hard to believe that so many statistical fluctuations could occur in the same mass, and it is tempting to conclude that a narrow resonance structure exists at a mass near $1535 \mathrm{MeV}$. However, let us take a critical look at all of the individual experiments.

\subsection{The first four experiments}

First results from the LEPS 1], DIANA [2, CLAS 3] and SAPHIR 4] collaborations were ground-breaking, but each experiment has some weakness. The LEPS experiment had only 19 counts in the peak on top of a background that was 17 counts, so detailed studies of the systematic uncertainties become very difficult. The final plot from the LEPS data is shown in Fig. 8, where the 


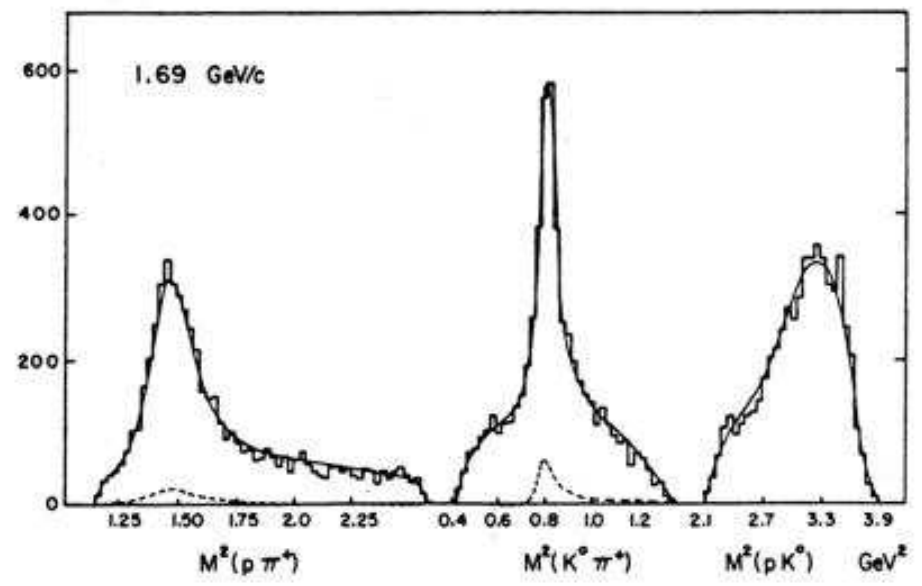

Figure 6: Data of Berthon et al. [54] for $K^{+}$particles of momentum $1.69 \mathrm{GeV} / \mathrm{c}$ incident on a hydrogen bubble chamber. If the pentaquark has mass $1.54 \mathrm{GeV}$ then it would appear as a peak at $M^{2}=2.37 \mathrm{GeV}^{2}$ in the $p K^{0}$ invariant mass spectrum on the right. 


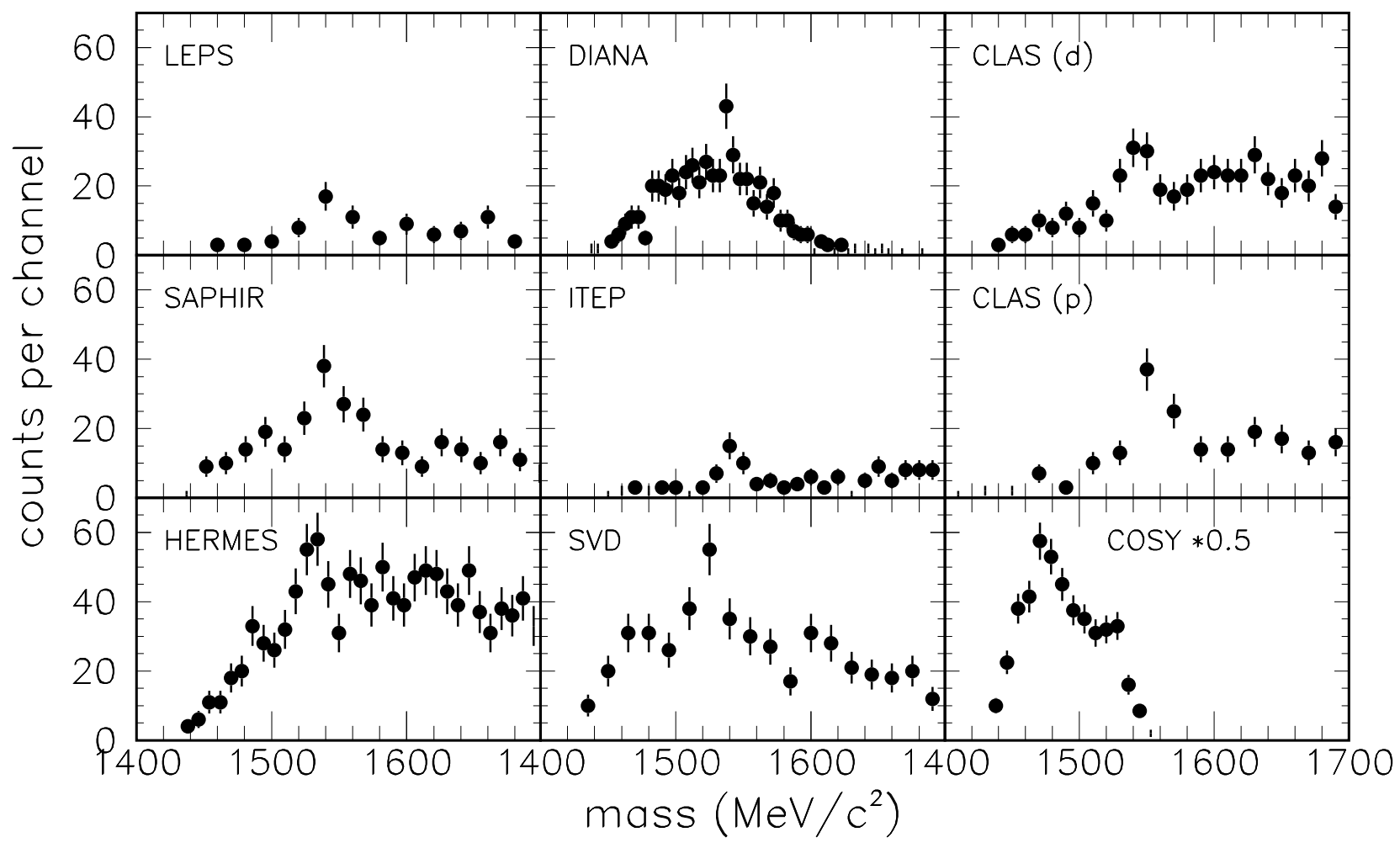

Figure 7: Data from experiments with positive evidence for the $\Theta^{+}$(see Table 1) taken from Ref. [57]. 

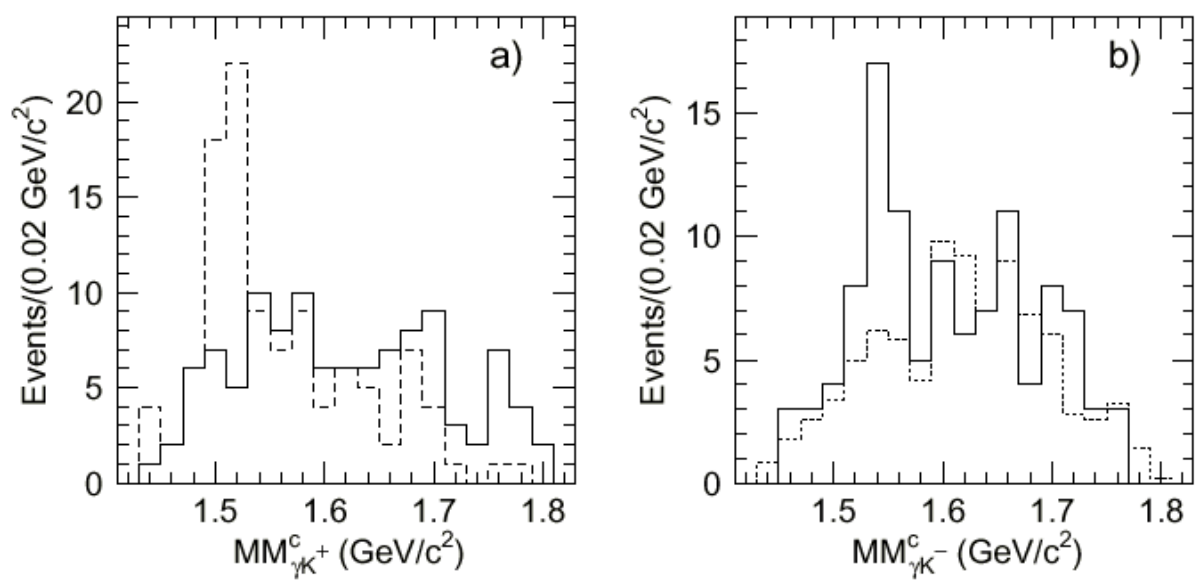

Figure 8: Data from LEPS [1] for the reaction $\gamma C \rightarrow K^{+} K^{-} X$. Missing mass spectra for the $K^{+}$and $K^{-}$corrected for Fermi motion are shown. The solid histogram shows events where no proton was detected. The $\Lambda(1520)$ resonance is seen on the left in the dotted histogram, where a coincident proton was detected. Possible evidence for the $\Theta^{+}$is seen by the peak on the right.

missing mass (corrected for Fermi motion) of each kaon in the $\gamma C \rightarrow K^{+} K^{-} X$ reaction is shown, along with events where a recoil proton has been detected (dashed histogram). A recoil proton is often detected in reactions where the proton was struck, such as the $\gamma p \rightarrow K^{+} \Lambda(1520)$ reaction, followed by the decay $\Lambda^{*} \rightarrow p K^{-}$. Hence the $\Lambda(1520)$ peak is seen in the dashed histogram on the left. On the other hand, reactions on the neutron, such as $\gamma n \rightarrow K^{-} \Theta^{+}$ followed by the decay $\Theta^{+} \rightarrow n K^{+}$, will not have an energetic proton. Hence the peak in the solid histogram on the right is interpreted as the $\Theta^{+}$and the dashed histogram shows a possible background from quasi-free production of kaon pairs, which can happen on either protons or neutrons. Note that the LEPS detector has a forward-angle-only acceptance, and that it is symmetric for detection of positive and negative particles, which is helpful when comparing the $\Lambda(1520)$ and $\Theta^{+}$peaks.

Several questions occur when examining these data. First, how is the correction for Fermi motion done? The answer is that it is an empirical correction which is an approximation, good only if the momentum transfer to the residual nucleus is small. One must be careful of approximations when claiming evidence for a new particle. In addition, how well is the background determined? Is quasi-free production the dominant process, or are there other secondary scattering reactions that could "reflect" into the phase space at the region of the peak? If one chooses a different background that has a shape which is higher in the region of the possible $\Theta^{+}$peak, then the evidence becomes much weaker. Without more details of the proton veto efficiency and other systematic uncertainties in the background shape (which are not described in the LEPS paper [1]) then one must take a cautious attitude about this evidence for the $\Theta^{+}$. 
The mass spectrum from the DIANA experiment [2] is shown in Fig. 9] Here the reaction is $K^{+} X e \rightarrow K^{0} p X e^{\prime}$ followed by the decay $K_{s}^{0} \rightarrow \pi^{+} \pi^{-}$. The final-state $p \pi^{+} \pi^{-}$particles are detected by ionization tracks in the Xe bubble chamber photographs. The final data sample has cuts on the proton and $K^{0}$ emission angles (both are required to be $<100^{\circ}$ in the lab frame, and their azimuthal angles must be at least $90^{\circ}$ apart) in order to remove rescattering events. (The DIANA experiment is hampered by background from kaon charge-exchange reactions.) Not enough detail is given in their paper to show how the cuts they employ affect the mass spectrum where the $\Theta^{+}$peak is seen, which is concentrated into a single bin. In fact, if one looks at the mass spectrum before these angle cuts, there is only the slightest hint of a peak.

One advantage of the DIANA experiment is that there is little chance of particle misidentification based on the kinematic constraints to form a $K^{0}$ peak from a $\pi^{+} \pi^{-}$pair. Another advantage is that the hadronic reaction mechanism conserves strangeness, and has the same quantum numbers in the initial state as for the $\Theta^{+}$. Also, the mass spectrum is calculated from the invariant mass of the $p K^{0}$ system, and so a "mixed-event" technique can be used to estimate the background (shown by the dotted histogram in the figure) where protons and $K^{0}$ 's from different events are combined together randomly. This technique has been used by many experiments in the past as a reliable way to estimate the background shape.

The main disadvantage of the DIANA experiment is that there are no details on the sensitivity of the mass spectrum to the angle cuts, nor are there details on the modeling of the charge-exchange background (just some general comments that simulation studies were done 2]). One must be extremely cautious about peaks that appear only after angle cuts are made, and especially if the peak comes all in one bin of the histogram. They estimate the statistical significance of their peak to be $4.4 \sigma$, but a simple calculation shows that the single channel is only 3 standard deviations from their estimated background. The combination of arbitrary angle cuts and small statistical significance suggests that this result, taken alone, is not convincing evidence for a new particle.

The CLAS data 3 was the first exclusive reaction, using the reaction $\gamma d \rightarrow$ $K^{+} K^{-} p(n)$ on a deuterium target. The neutron was not detected directly, but deduced from the missing 4-momentum, with very little background $(<15 \%$ under the neutron mass peak). However, detecting the proton requires it to have a momentum well above the Fermi momentum $(>300 \mathrm{MeV} / \mathrm{c})$ and hence the proton cannot be a spectator. Since the $\Theta^{+}$can only be produced (in this final state) on the neutron there must be a secondary reaction, such as rescattering of the $K^{-}$from the proton, which complicates the reaction diagram. One might expect that the probability of rescattering would be low, but in fact it can be shown to happen with about $30-50 \%$ probability for $\Lambda(1520)$ production.

As a result, the shape of the background under the $\Theta^{+}$peak is difficult to estimate and may include kinematic reflections [58. (Note that the mechanism suggested by Ref. [58] has been challenged because it violates C-parity conservation, see [59.) However, it is true that the shape of the background is unknown, and the CLAS result only becomes significant if one accepts the background shape given in their paper [3]. An alternative background that 


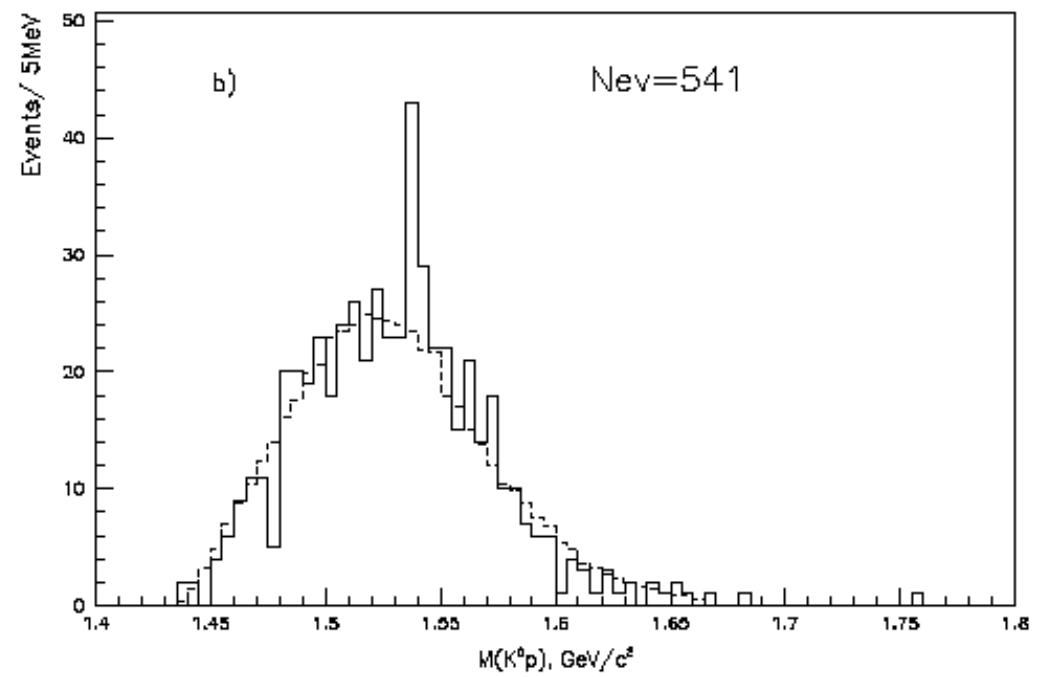

Figure 9: Data from DIANA [2] for the reaction $K^{+} X e \rightarrow p K_{s}^{0} X e^{\prime}$. The invariant mass of the $p K^{0}$ system is shown after analysis cuts (see text). The dotted histogram is from a mixed-event technique expected to represent the background.

stays higher, cutting through the middle of the peak in Fig. 10] and then drops sharply at lower mass would reduce the statistical significance to about $3 \sigma$, rather than the $5.2 \sigma$ for the background shape used in their paper. Again, taken alone, this results could potentially be the result of an unlucky statistical fluctuation.

The SAPHIR collaboration was the first to publish results for the exclusive $\gamma p \rightarrow K_{s}^{0} K^{+} n$ reaction, which does not require any rescattering or any nuclear effects. In principle, this is the best reaction to provide convincing evidence that the $\Theta^{+}$exists. Their mass spectrum is shown in Fig. 111, after subtraction for background on either side of their $K^{0}$ peak (not shown, see Ref. [4]). In addition, an angle cut on the $K^{0}$ requiring it to be only at forward angles (center-of mass angle with $\cos \theta_{K^{0}}>0.5$ ) has been applied. The peak appears to be substantial (about 4 sigma), however the large cross section they estimated from their measurement conflicted with data for the same reaction measured at CLAS 60. Of course, CLAS does not have the same detector acceptance as SAPHIR, and if the $\Theta^{+}$is produced at forward angles then SAPHIR has an advantage. Still, a reanalysis of the SAPHIR data 61] suggests a smaller cross section (this revised analysis result has not been published).

As I write, the CLAS collaboration is preparing to release high-statistics results on the same reaction measured by SAPHIR. The result is simply a flat mass spectrum, regardless of the region of the $K^{0}$ angle. This new result is in direct contradiction to the SAPHIR data. One can question whether the $\Theta^{+}$can be produced on the proton (for example, the diagram given in Ref. [4] cannot contribute due to C-parity violation). In fact, most calculations 63, 62 show that production cross sections on the neutron are predicted to be much larger than those for the proton, although the calculations at present are very model-dependent 64. In any case, the high-statistics result of CLAS 


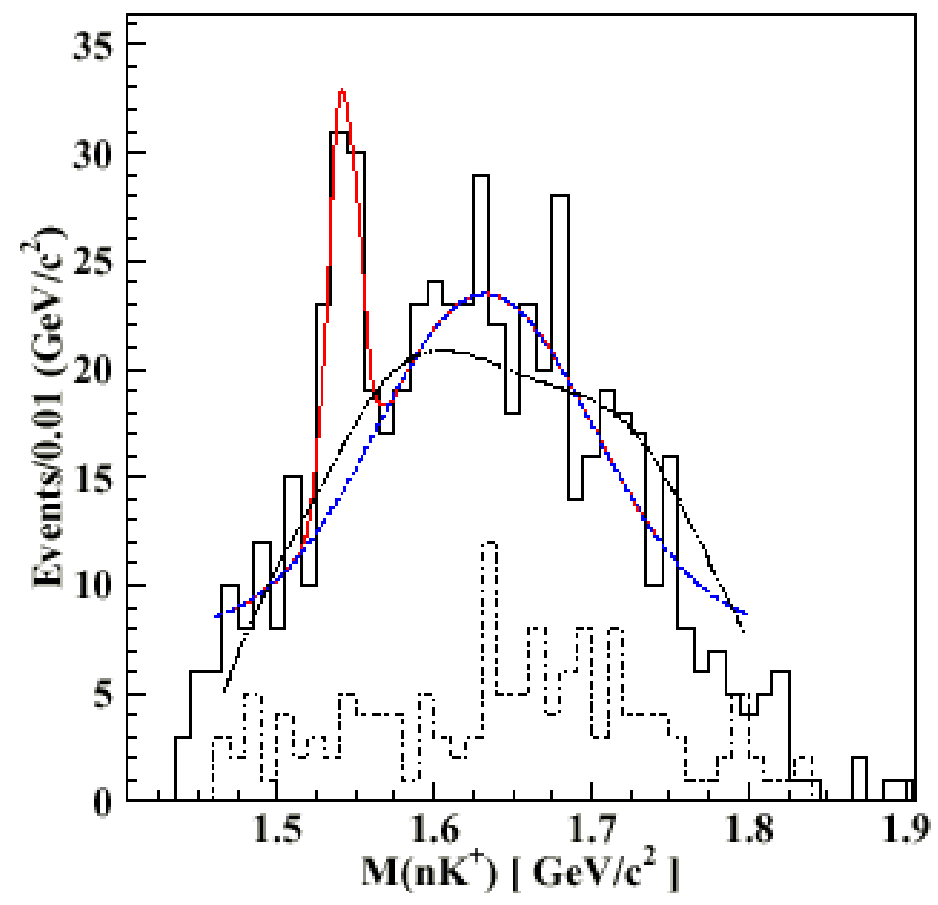

Figure 10: Data from CLAS [ 6 for the reaction $\gamma d \rightarrow K^{+} K^{-} p(n)$. The missing mass of the $K^{-} p$ system, which is the same as the mass of the $n K^{+}$system, is shown along with two estimates of the background shape (smooth curves). The contribution of the $\Lambda(1520)$ events, which were cut out, are shown by the dashed histogram at the bottom. 


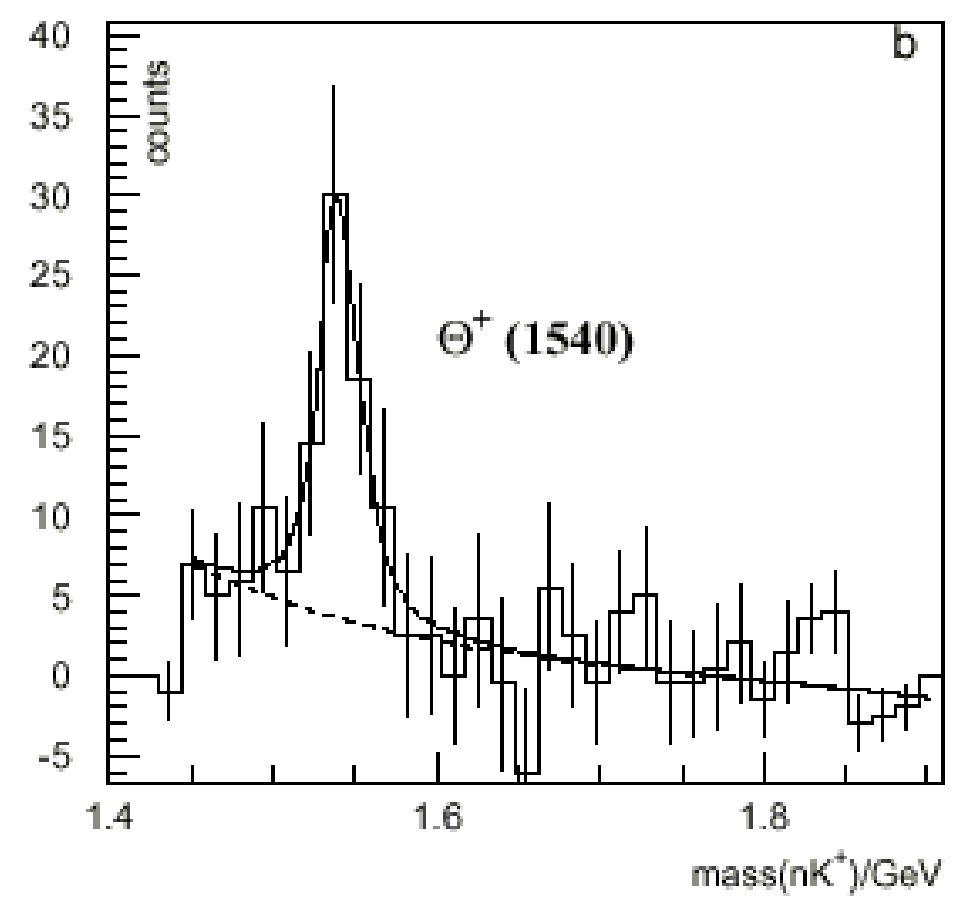

Figure 11: Data from SAPHIR [4] for the reaction $\gamma p \rightarrow K^{+} K_{s}^{0}(n)$. The mass of the $n K^{+}$system is shown, after sideband subtraction on either side of the $K^{0}$ peak and cuts on the $K^{0}$ angle (see text).

shows that $\Theta^{+}$production in the $\gamma p \rightarrow K_{s}^{0} K^{+} n$ reaction is, at the least, highly suppressed.

\subsection{The $p K^{0}$ experiments}

Following the first reports, several experiments measured the invariant mass of the $K_{s}^{0}$ and a proton, from inclusive production. One of these collected data from neutrino experiments (ITEP [5]), two others used electroproduction ( HERMES [7] and ZEUS 8]) and another used a proton beam (SVD [10]). Of course, the $K_{s}^{0}$ is a mixture of both strangeness +1 and -1 , so the invariant mass spectra will include both $\Sigma^{*+}$ and possible $\Theta^{+}$peaks. It follows that a peak at a mass where no $\Sigma^{*+}$ resonance is known could be evidence for the $\Theta^{+}$ or an unknown $\Sigma^{*+}$ resonance. It is also curious that these four measurements reported a $\Theta^{+}$mass which is about $10 \mathrm{MeV}$ below that seen by the first experiments (barely compatible within the experimental uncertainties). The inherent weakness of not knowing the strangeness of a particle, coupled with the uncertainty in the background which must include the overlapping $\Sigma^{*+}$ resonances, makes this evidence less convincing than exclusive measurements. Next the details will be discussed.

The ITEP group [5] analyzed a conglomerate of five neutrino experiments 


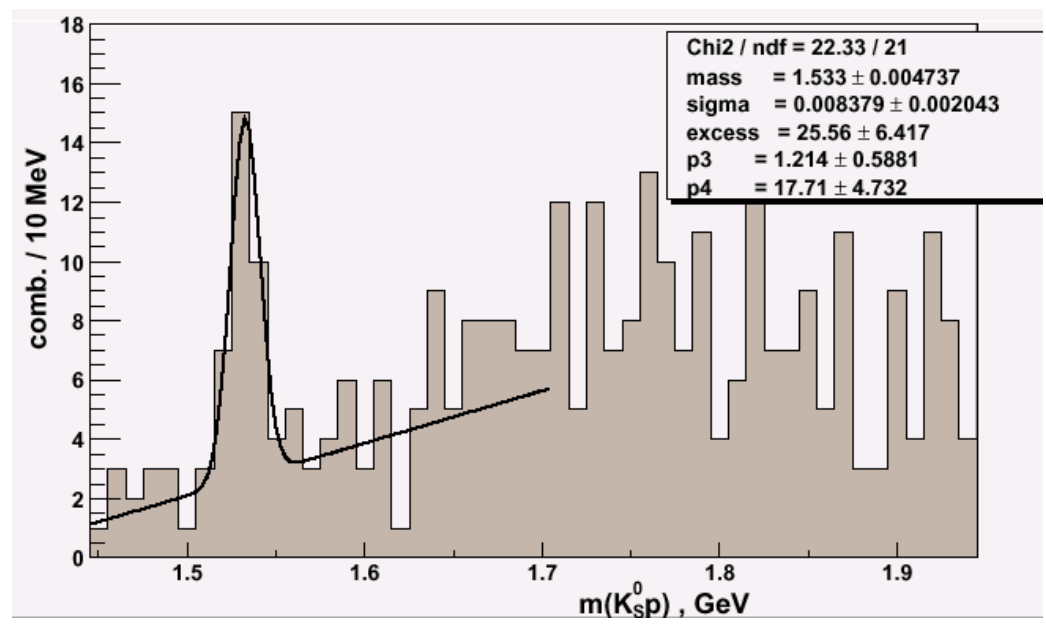

Figure 12: Data from ITEP [5] for the reaction $\nu A \rightarrow p K_{s}^{0} X$ where A represents a sum of D and Ne bubble chamber data.

from bubble chambers using the $\nu A \rightarrow p K^{0} X$ reaction, where $A$ represents either hydrogen $(\mathrm{H})$, deuterium $(\mathrm{D})$, or neon $(\mathrm{Ne})$. In these data, the statistics are very low for $\mathrm{H}$ and $\mathrm{D}$, so essentially all of the peak shown in Fig. 12 comes from the Ne target. (Their result is actually extracted by adding together the $\mathrm{Ne}$ and $\mathrm{D}$ data.) The background can be estimated by taking random combinations of protons from one event and $K^{0}$ 's from another event, which must be uncorrelated and hence gives a smooth background, which is similar to the DIANA background analysis. The background shown in Fig. 12 is just a linear fit to the background, whereas the random (combinatorial) background is a bit higher [5]. There are about 20 counts above a background of 12 counts, giving a realistic statistical significance of about $3.5 \sigma$ (although they claim 6.7 $\sigma)$ at a mass of $1533 \mathrm{MeV}$.

The advantages of the ITEP measurement are: (1) low background and (2) a quantitative combinatorial background. The disadvantages are: (1) the strangeness of the $K^{0}$ is unknown and (2) low statistics. In addition, there is no confirmation by modern neutrino experiments (although this could change in the near future), and a strong peak like this should clearly be visible at higher statistics. Only time will tell if this peak is real or yet another coincidental statistical fluctuation.

The HERMES collaboration measured the $e^{+} d \rightarrow p K^{0} X$ reaction using the DESY accelerator positron beam at $27.6 \mathrm{GeV}$ onto a stationary deuterium target. Their event selection, based on particle identification of in a RICH detector [7, was optimized for the $p K^{0}$ final state resulting in a clear peak for the $K^{0}$ in the $\pi^{+} \pi^{-}$invariant mass spectrum. Combined with the proton, the $\pi^{+} \pi^{-} p$ invariant mass spectrum is shown in Fig. 13] along with a fit to the peak and background. They also tried other backgrounds based on simulations (using PYTHIA6) along with fits to known $\Sigma^{*+}$ resonances at higher mass. They also tried a different method for the invariant mass where the $K^{0}$ mass is used as an explicit constraint. Depending on the various fits, the statistical significance of the peak (based on fitting errors) is between 3.4-4.3 $\sigma$. 


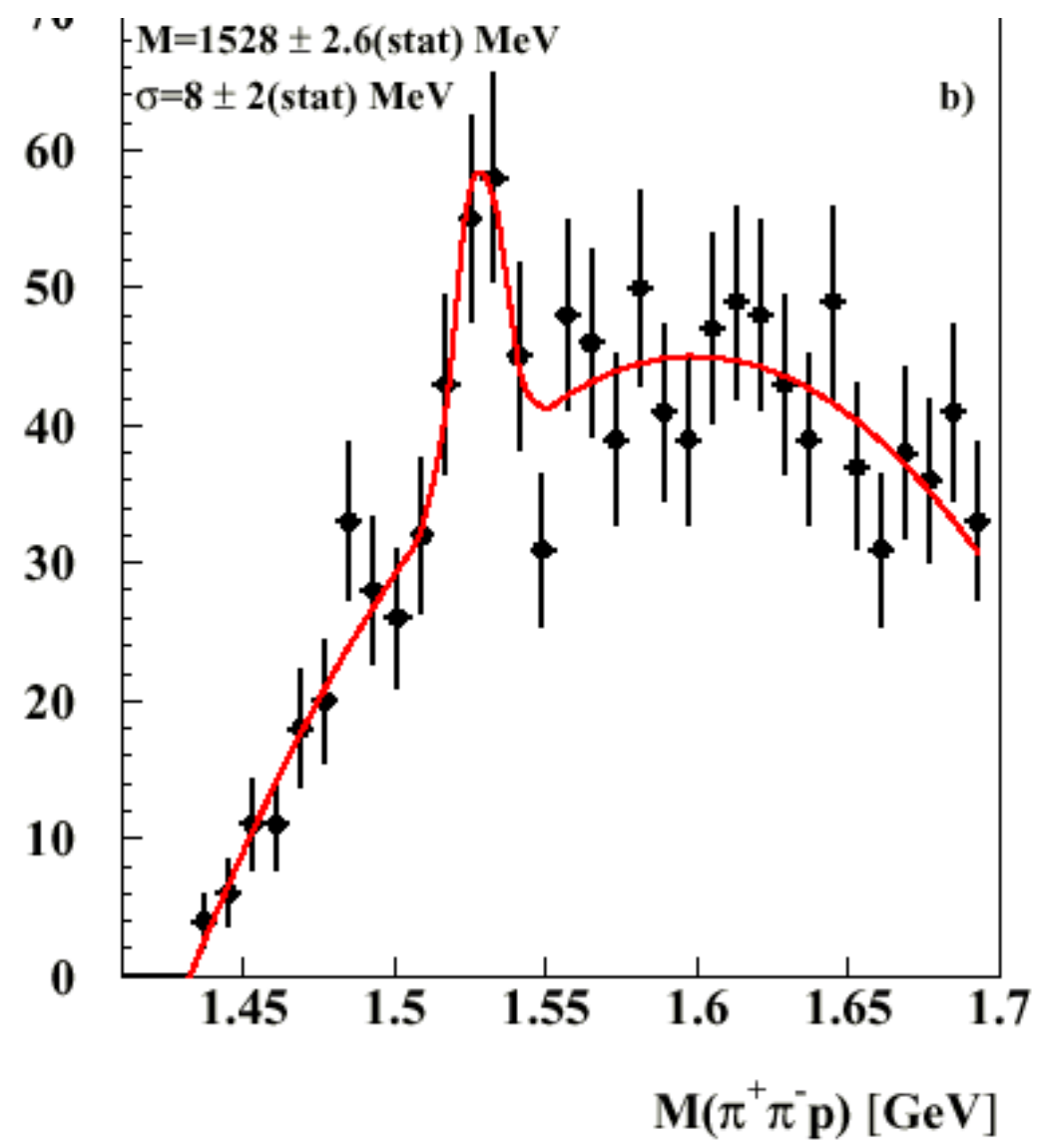

Figure 13: Data from HERMES [7] for the reaction $e^{+} d \rightarrow \pi^{+} \pi^{-} p X$ along with a fit to the peak and a smooth background.

One of the major concerns with this result is that it is lower in mass at $1528 \mathrm{MeV}$ with a small error $(2.6 \mathrm{MeV})$. This is clearly inconsistent with the DIANA experiment which also measured the $p K^{0}$ invariant mass at $1539 \pm 2$ $\mathrm{MeV}$. How can both results be the same particle, unless there are large systematic uncertainties in one experiment (or both)? A second concern is that the HERMES experiment does not have a strangeness tag (unlike DIANA), and so the peak could in principle be either the $\Theta^{+}$or a new $\Sigma^{*+}$ resonance. However, recent reanalysis of the HERMES data [65] suggests strongly that it is unlikely to be $\Sigma^{*+}$ resonance. Also in Ref. 65 the analysis was extended to remove events where an extra pion, not part of the $K^{0}$ decay, was detected and can be shown to be part of $\phi$ or $\Lambda$ production. This method gives a better signal to background ratio, but also has lower statistics. It would be interesting if more data could be taken, but this is unlikely to happen in the near future.

The ZEUS experiment 8 did a similar measurement, also at DESY, for the $e^{+} p \rightarrow K^{0} p X$ reaction except now at a center-of-mass energy of about $300 \mathrm{GeV}$. At such high energies, hadrons are produced primarily through fragmentation. The fragmentation process in the deep inelastic scattering (DIS) region is thought to proceed via "string-breaking", where one quark is given almost all of the energy transfer from the scattered lepton followed by multiple 
breaking of the color-force flux-tube that connects the outgoing quark to the residual diquark. As explained below, fragmentation is an unlikely method to produce the $\Theta^{+}$(or any multi-quark object). Nonetheless, the ZEUS result, shown in Fig. 14 shows a clear peak near $1522 \mathrm{MeV}$ when they take events with 4-momentum transfers $Q^{2}>20 \mathrm{GeV}^{2}$. In this figure, both $K^{0} p$ and $K^{0} \bar{p}$ data have been added together, where the latter is for the anti- $\Theta^{+}$since the $K_{s}^{0}$ is an equal mixture of both $K^{0}$ and anti- $K^{0}$ states.

The same question applies to ZEUS as it did to HERMES: how can the mass of their peak be consistent with the earlier experiments? Of course, one could argue that many of those experiments had large systematic uncertainties on the mass, but even the ZEUS and HERMES results disagree in mass by 6 $\mathrm{MeV}$ (more than two standard deviations). Furthermore, why is the selection criteria $Q^{2}>20 \mathrm{GeV}^{2}$ necessary? The ZEUS data is also shown for $Q^{2}>1$ $\mathrm{GeV}^{2}$ [8] and no peak is visible. These questions are disturbing, especially if one tries to explain away the null results (see below) based on suppression of the $\Theta^{+}$production via fragmentation. In other words, if ZEUS sees a $\Theta^{+}$peak, then other high-energy experiments (where fragmentation dominates) should also see a $\Theta^{+}$peak.

Could the ZEUS peak possibly be due to a statistical fluctuation? Using simulations (with the ARIADNE package) they are able to estimate their background shape and then fit the $\Theta^{+}$peak along with a possible lower-mass $\Sigma(1465)$ state, giving $221 \pm 48$ events for a statistical significance of $4.6 \sigma$. They also did a fit without the $\Sigma^{+}$state, getting only $3.9 \sigma$. In addition, they did a fit to the entire spectrum without any peaks, and used this to generate Monte Carlo spectra, and found the probability to make a peak, with at least $3.9 \sigma$ somewhere in the mass range of 1500 to $1560 \mathrm{MeV}$, to be $6 \times 10^{-5}$. It seems unlikely that this peak is a statistical fluctuation.

On the other hand, it is difficult to see how the ZEUS peak can be the $\Theta^{+}$unless many other experiments are wrong. Why is the ZEUS mass so much lower than the other experiments? Why is the $\Theta^{+}$not seen in other experiments where fragmentation dominates? Until these questions can be answered, it seems best to wait for higher statistic from ZEUS from their new data set (currently being analyzed). A detailed discussion of the pentaquark searches at HERA are give in Ref. [66].

The SVD collaboration [10] measured the $p A \rightarrow p K^{0} X$ using the $70 \mathrm{GeV}$ proton beam at the IHEP accelerator in Russia. Their result, the invariant mass of the $p K^{0}$ system, is shown in Fig. [15] using events with no more than 5 charged tracks and a requirement that the angle of the $p K^{0}$ system be forward of $90^{\circ}$ in the center-of-mass. There is an excess of counts at about $1526 \mathrm{MeV}$ above their background estimated by FRITIOF simulations. In addition, there is excess strength in the $1570-1750 \mathrm{MeV}$ range, presumably due to higher-mass $\Sigma^{*+}$ resonances. Without true knowledge of the background, it is difficult to estimate the statistical significance, but it is likely about $3 \sigma$ (or less).

An additional cut, where the momentum of the $K^{0}$ is required to be less than the momentum of the proton, suppresses the $\Sigma^{*+}$ resonances (as expected based on kinematics of their decay) while having little effect on the proposed $\Theta^{+}$peak. With this cut, the $\Theta^{+}$peak in their mass spectrum looks better 


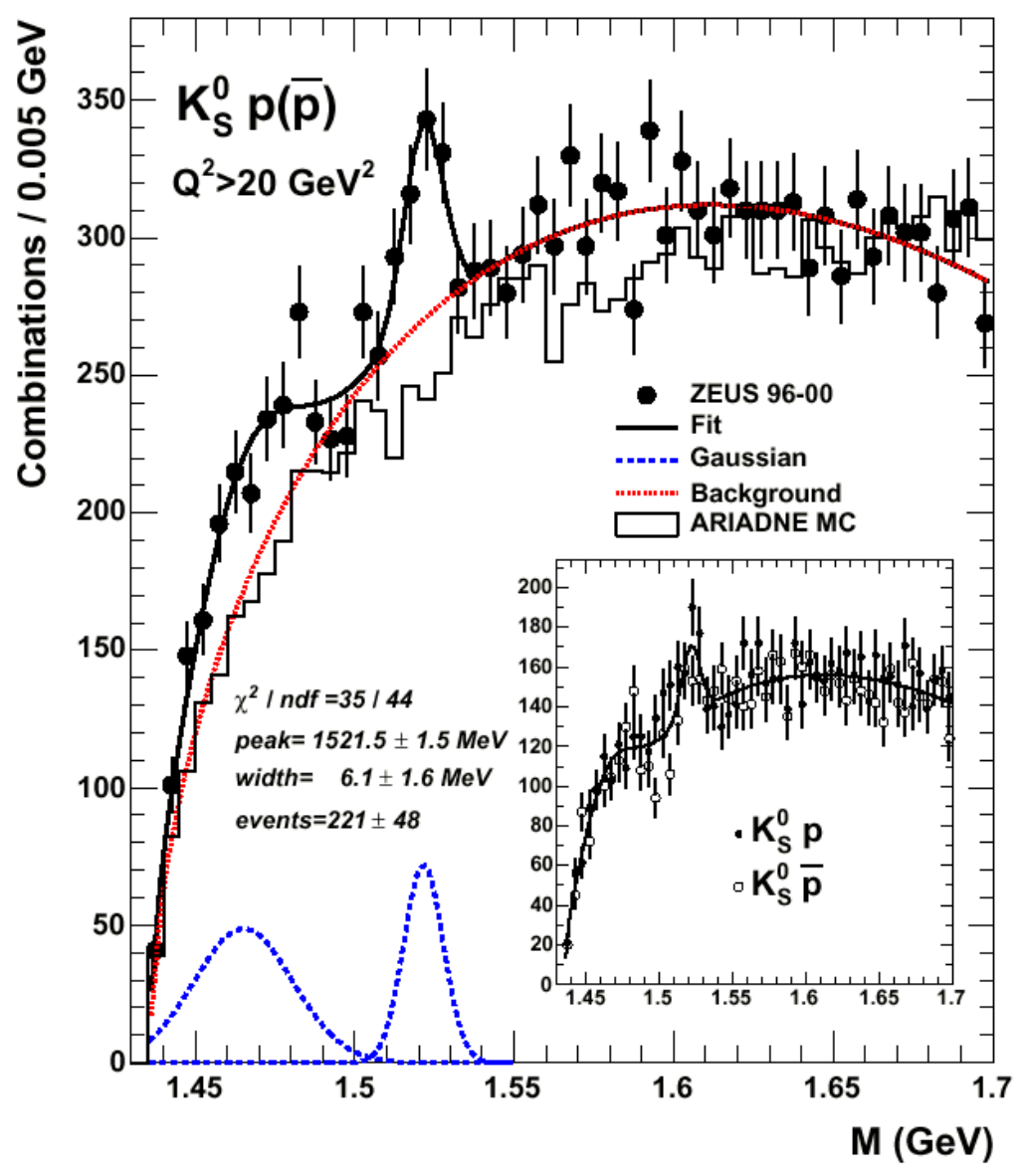

Figure 14: Data from ZEUS [8 for the reaction $e^{+} p \rightarrow K^{0} p X$ with a cut on the 4 -momentum transfer $Q^{2}>20 \mathrm{GeV}^{2}$. The solid line is a fit to a smooth background and two peaks: a known $\Sigma^{*+}$ resonance and a possible $\Theta^{+}$peak at $1522 \mathrm{MeV}$. A Monte Carlo background is also shown by the histogram. The inset shows the two separate event sets added together in the main figure. 


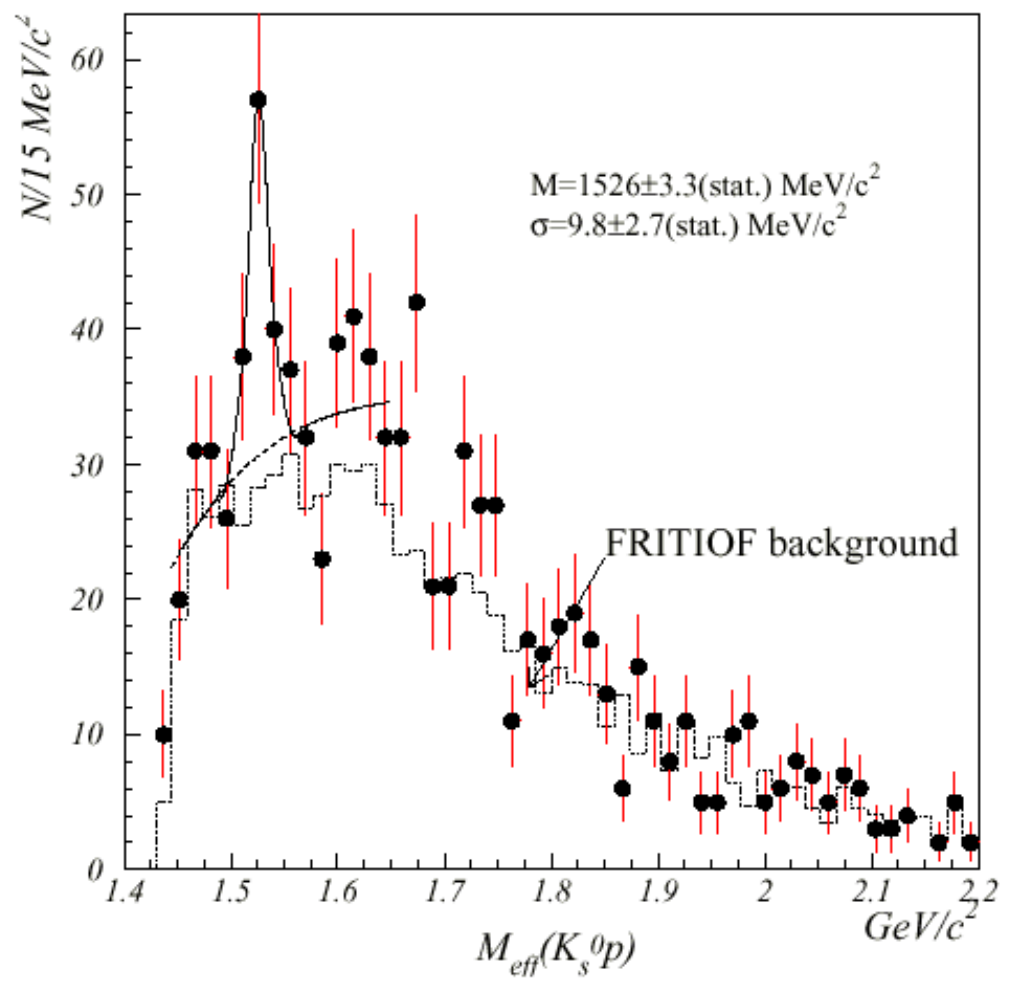

Figure 15: Data from SVD [10] for the reaction $p A \rightarrow p K_{s}^{0} X$ when the $p K^{0}$ system is forward of $90^{\circ}$ in the center-of-mass frame. An estimate of the background is shown by the FRITIOF simulation.

[10], but this kind of kinematic cut should be regarded with caution, since the kinematics of $\Theta^{+} \rightarrow K^{0} p$ is not very different from $\Sigma^{*+} \rightarrow K^{0} p$ decay.

The mass of their peak is closer to that measured by HERMES, but still consistent with ZEUS. So we have the rather paradoxical situation that the latter four experiments, all detecting the $p K^{0}$ system with no strangeness tag, have their peaks at lower mass than the $\Theta^{+}$peaks in the first four experiments, where the detection of the $K^{+}$tags the strangeness to ensure that their spectra had strangeness $S=+1$. Although it seems unlikely that there is an unknown $\Sigma^{*+}$ state near $1525 \mathrm{MeV}$, this possibility cannot be ruled out. In any case, it is not clear that the latter four experiments support the case for the $\Theta^{+}$ because of this discrepancy in the masses of the peaks. Note that this is not the first time where the masses of new particles "jumped around", as shown by the history plots in the Review of Particle Properties [69. Nonetheless, the movement of the peaks coupled with the low statistics should make one wonder about the strength of the positive evidence.

\subsection{The best positive evidence}

Two experiments have good evidence for the $\Theta^{+}$. The first is from CLAS on a proton target [6]. This exclusive reaction, $\gamma p \rightarrow \pi^{+} K^{-} K^{+} n$ is very clean, and the background comes primarily from meson production reactions. The 
results are shown in Fig. 16 where cuts have been applied on the $\pi^{+}$angle $\left(\cos \theta_{\pi}>0.8\right)$ and $K^{+}$angle $\left(\cos \theta_{K^{+}}>0.6\right)$ in the center-of-mass frame. The cuts for this analysis were not chosen arbitrary, as has been suggested by some critics, but are specifically designed to remove the dominant background (vector meson production) using the assumption that the $\Theta^{+}$can be produced through an s-channel diagram [6]. Furthermore, these data were subjected to a partial wave analysis (PWA), where the amplitudes of each partial wave were fit over the full angular coverage of the CLAS detector. Hence, the background under the $\Theta^{+}$peak (after all cuts are applied) has been fixed by the PWA from the full (uncut) data and is represented by the smooth curve under the peak.

The $\Theta^{+}$peak here is quoted as having the highest statistical significance yet, in excess of $7 \sigma$, although a more realistic estimate is perhaps $4 \sigma$. Because this is an exclusive measurement from the proton, there is no ambiguity of rescattering from other nucleons, and the strangeness of the final state is known. The authors have also checked using simulations of phase space, $t$-channel vector meson production, and data outside the cuts that a false peak is not artificially generated by the detector acceptance or from sensitivity to the analysis cuts.

On the other hand, the mass of the peak is at $1.55 \pm 0.01 \mathrm{GeV}$, which is about $0.01 \mathrm{GeV}$ higher than the first four experiments and about $0.02 \mathrm{GeV}$ higher than the $p K^{0}$ experiments. If the CLAS proton result is correct, then it is incompatible with the HERMES, ZEUS and SVD result. So although the CLAS proton results appear to be reasonably sound, the interpretation of the peak as the $\Theta^{+}$is questionable since the mass is so different from all other experiments.

Another experiment with good evidence for the $\Theta^{+}$is the COSY-TOF result [9] from the exclusive hadronic reaction $p p \rightarrow \Sigma^{+} K_{s}^{0} p$. Their results are shown in Fig. 17which is the sum of two data sets (taken in different years with slightly different beam energy) after acceptance corrections have been applied. Note that this figure is a bit different than that shown in the montage (Fig. 7) which shows the same data before acceptance corrections. Here, the strangeness of the $p K_{s}^{0}$ invariant mass is tagged by the $\Sigma^{+}$. The particle identification is done entirely by geometric reconstruction which, for this near-threshold reaction, is very accurate. Some critics have questioned whether this method provides good identification of the final state, but it can be rigorously proved that the kinematics are over-constrained [9]. The result is a very clean final state showing a $\Theta^{+}$peak at a mass of about $1.53 \mathrm{GeV}$, which is in the middle of the $\Theta^{+}$mass measurements, with a statistical significance of between 4 to $5 \sigma$ (depending on the background shape).

One problem with the COSY-TOF data is that the broad hump near threshold (maximum at about $1.47 \mathrm{GeV}$ ) is unexplained. Could this be yet another unlucky coincidence where the background appears to go smoothly under the peak but in reality the shape is much higher in the region of the peak? Only higher-statistics (or a quantitative calculation of the background) will tell us if this peak is real. The COSY-TOF collaboration has taken more data in Nov.Oct. 2004, which is expected to increase their statistics by a factor of about 5 [67, with a slightly increased beam energy (so that the peak is not so close to the end-point mass. This is really the only way to address whether the peak is 


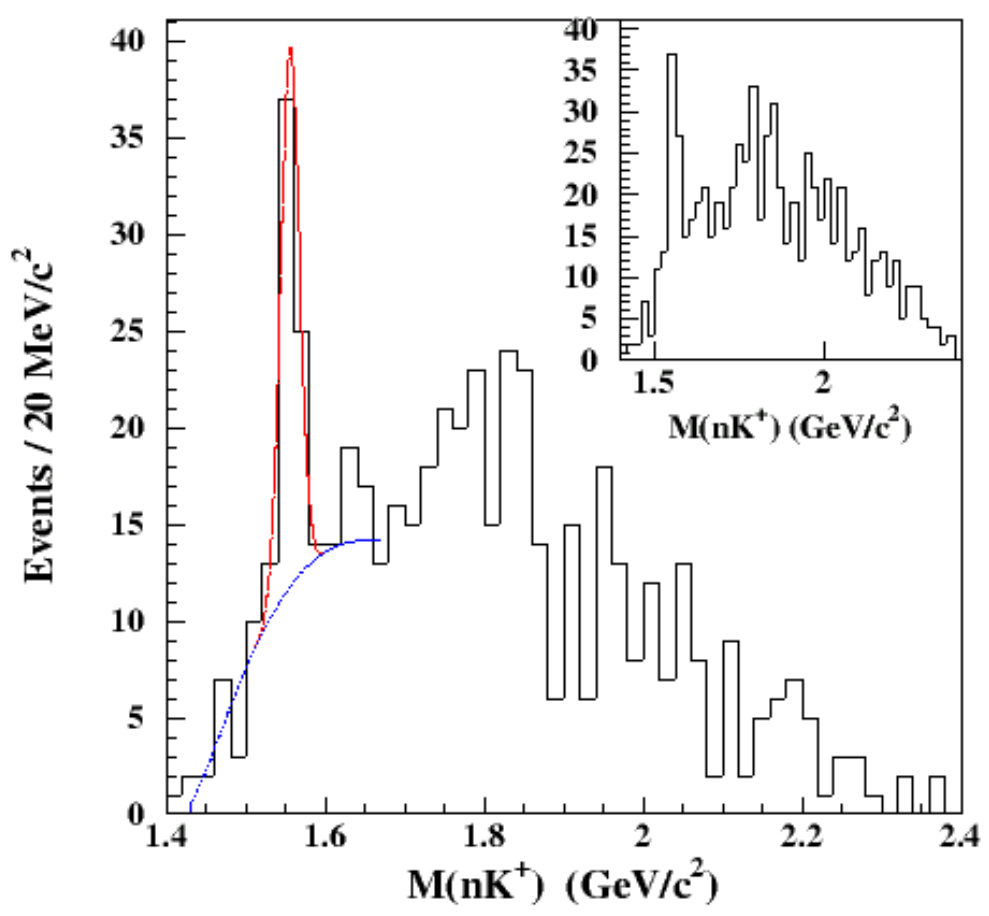

Figure 16: Data from CLAS [6] on a proton target for the reaction $\gamma p \rightarrow \pi^{+} K^{+} K^{-}(n)$ after cuts on the $K^{+}$and $\pi^{+}$angles. The inset shows the data when only the $\cos \theta_{\pi}>$ 0.8 cut has been applied. The curve is a fit to the peak and a smooth background estimated from partial wave analysis of the uncut data. 


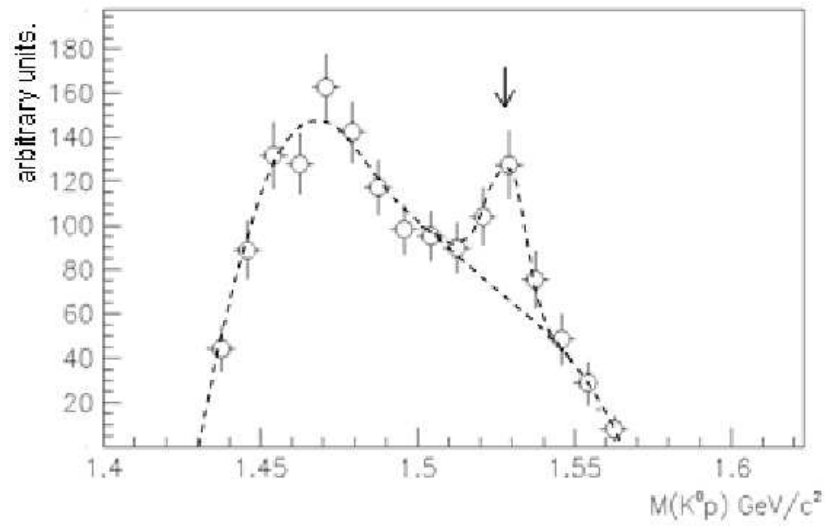

Figure 17: Data from COSY-TOF [9] for the reaction $p p \rightarrow \Sigma^{+} K_{s}^{0} p$ at near-threshold energies. The invariant mass of the $K^{0} p$ system is shown after acceptance corrections.

real, by gathering much higher statistics. Until then, patience and caution are advised.

\section{Non-observation experiments}

Having taken a critical look at the evidence in favor of the $\Theta^{+}$we now turn to the null results. These results come from either electron-positron colliders (BES 11], BaBar [12, Belle [13], LEP 14]) or from high-energy reactions using a hadron beam (such as HERA-B [15], SPHINX [16], HyperCP [17] and CDF [18]). Because of the difficulty in detecting neutrons in these detectors, these experiments look at the $p K_{s}^{0}$ invariant mass. Unlike the previous experiments, the high-energy hadron beam experiments typically have higher statistics yet see no $\Theta^{+}$peak.

Naively, one might expect that if the $\Theta^{+}$exists, it should be produced in both high-energy experiments through fragmentation processes as the flux tube breaks when the struck quark exits the nucleon. This reasoning suggests that the high-energy experiments should see the $\Theta^{+}$(if it exists). However, there are some curious features of the null results that will be discussed below.

\subsection{The $e^{+} e^{-}$experiments}

First, let us examine the $e^{+} e^{-}$collider data. The real question here is: how can the $\Theta^{+}$can be produced? The initial system starts with zero quarks, and in the final state, there are at least 5 quarks and 5 antiquarks. This number of quarks is produced regularly at these facilities, but the reaction mechanism is thought to start from a quark-antiquark pair at the $e^{+} e^{-}$annihilation point, and other quarks are produced in fragmentation or "string breaking" as the quark-antiquark separate. In order to form a $\Theta^{+}$, there must be 4 quarks and one antiquark localized in space with small relative velocity, something that would be difficult to achieve from the fragmentation process. A theoretical estimate by Azimov [68] suggests that the BES result (see Table 2) is not likely 


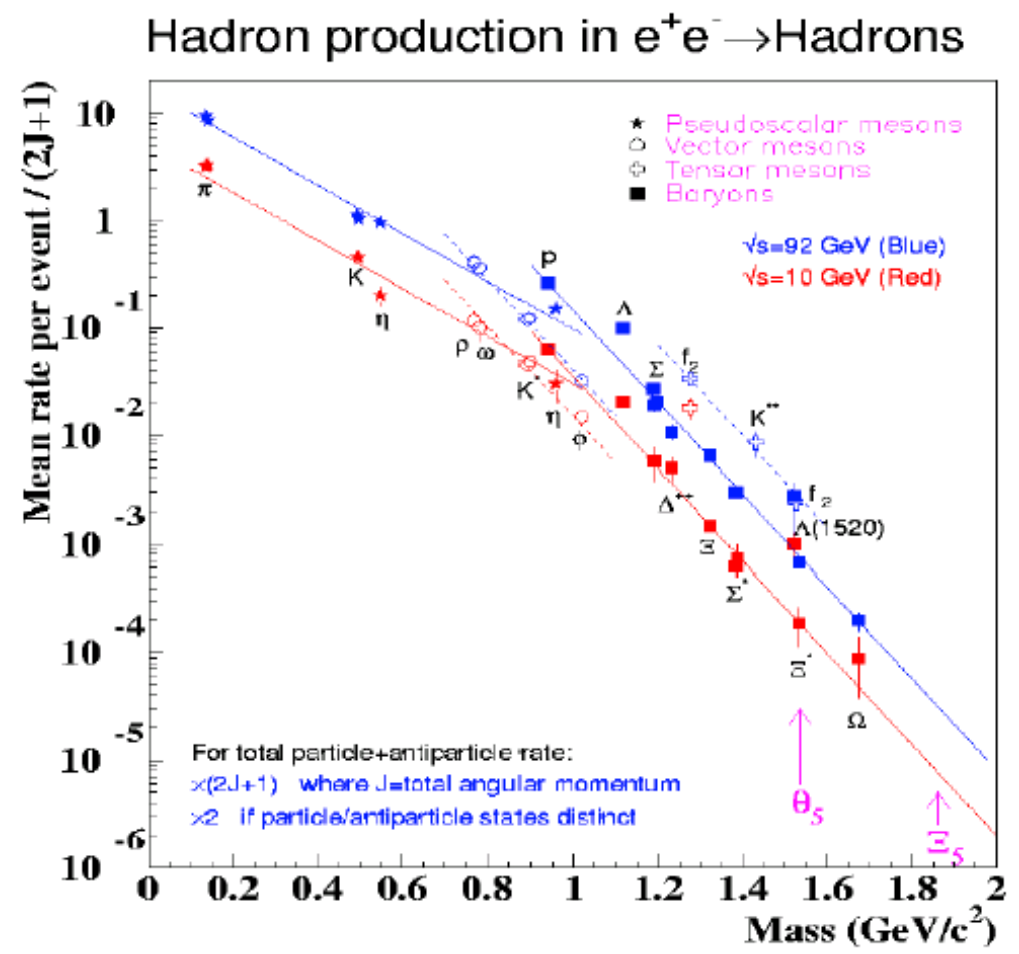

Figure 18: Hadron production rates from $e^{+} e^{-}$collisions at BaBar 12. The lower limits for $\Theta^{+}$and $\Xi^{--}$are shown by the arrows. Mesons are fit to a line at the left, and baryons are fit with lines having steeper slopes. Similar slopes are seen for center-of-mass energies of $10 \mathrm{GeV}$ (lower lines) and $92 \mathrm{GeV}$ (upper lines).

to be sensitive enough to search for the $\Theta^{+}$from $e^{+} e^{-}$collisions.

The BaBar collaboration found that the production of baryons follows a systematic trend where the probability of production decreases smoothly as the mass increases, see Fig. 18. (Note: the production rate is divided by $2 J+1$ where $J$ is the spin of the hadron.) In this figure, it is also shown that for mesons, the production rate versus mass is less steep than for baryons. The next question is: does the production rate falls off faster for pentaquark production? Unfortunately, there is no theory to guide us here, but the trend suggests that it would fall off faster, in which case these experiments may not have the sensitivity for a $\Theta^{+}$search, although this remains an open question.

The results from the BaBar search is shown in Fig. 19 for the invariant mass of protons and $K_{s}^{0}$ particles from inclusive $e^{+} e^{-}$reactions. The statistics here is huge, and the resolution is also expected to be good $(<2 \mathrm{MeV}$, which is the bin size of the points shown). No structure is seen in the mass range from $1525-1560 \mathrm{MeV}$. The data can also be divided in to subsets for smaller ranges of the momentum of the proton, and again no structure is seen. However, it is surprising that there are no structures, even broader peaks anywhere in the mass range! Since the strangeness of the $K_{s}^{0}$ is unknown, we might expect some $\Sigma^{*+}$ resonances (such as the $\Sigma(1670$ ) which has a width estimated at about 60 $\mathrm{MeV}$ 69]). In the plot to the right in Fig. 19 the data are extremely flat, 

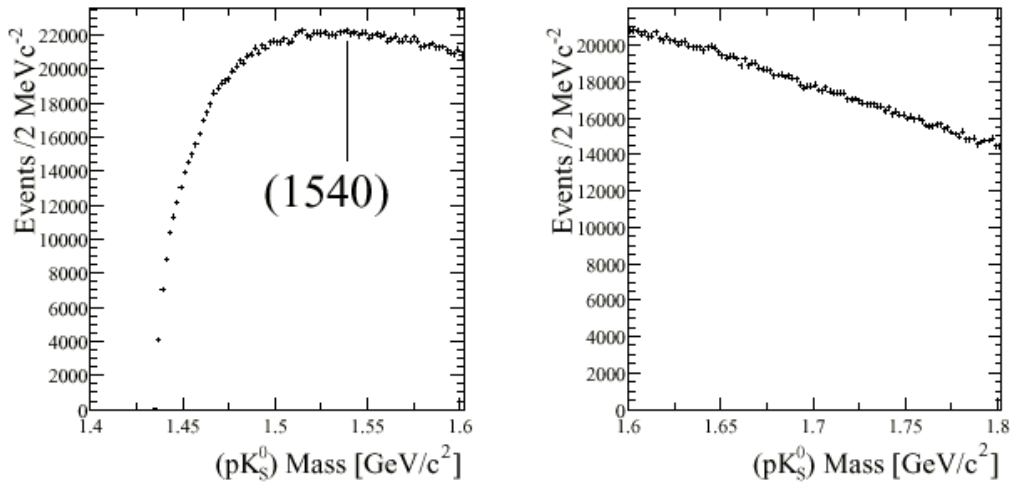

Figure 19: Data from BaBar 12] for inclusive events with a detected proton and $K_{s}^{0}$. No peak is seen near the $\Theta^{+}$mass nor are any broad resonances evident.

showing no signs of any of the known resonances. Of course, one can argue that these resonances are wide and overlapping, whereas the $\Theta^{+}$is expected to be quite narrow, but still it is surprising that there is no evidence of any structure, whereas the $\Lambda(1520)$ resonance (not shown) gives a large peak in the $p K^{-}$invariant mass spectrum.

The limit on pentaquark production from BaBar, given in Table 2, is useful information, but does not rule out that the $\Theta^{+}$pentaquark could exist. Similar comments apply to the Belle and LEP limits from $e^{+} e^{-}$production. Rather, there is an opportunity here to study the rate of hadrons produced in $e^{+} e^{-}$ collisions, with the goal to elucidate the reaction mechanism and study the spectrum of hadron masses from these beautiful data.

\subsection{High-energy hadron beam experiments}

Since the hadron beam experiments pose a more serious challenge to the existence of the $\Theta^{+}$we should examine these experiments with some care. In fairness, the same criticism directed at the $e^{+} e^{-}$experiments can also be applied to the high-energy hadron experiments, that the $p K_{s}^{0}$ spectra should show evidence for known $\Sigma^{*+}$ resonances.

The mass spectrum from the HERA-B experiment, which measured the $p C \rightarrow p K^{0} X$ reaction for protons at $920 \mathrm{GeV}$ onto a fixed Carbon target, is shown in Fig. 20. Again no peak is seen near $1540 \mathrm{MeV}$, nor is any structure seen throughout the spectrum. Note that here each data point has a bin width of about $10 \mathrm{MeV}$. Also, these data have higher particle multiplicities than for BaBar, which makes the combinatoric background (choosing which of several protons goes with the one or more $K_{s}^{0}$ particles) more difficult. The plot on the top right of Fig. 20 shows the reduced statistics when only events with multiplicity $<10$ are used, which should give a "cleaner" $p K^{0}$ mass spectrum.

The SPHINX experiment measured a similar reaction, $p C \rightarrow p K_{s} K_{L} X$ at 70 $\mathrm{GeV}$ (about a factor or 10 lower in beam energy than HERA-B), except that now they detected a coincident $K_{L}$ as a neutral cluster in their calorimeter. Their resulting spectra are shown in Fig. 21 for the invariant mass of the 


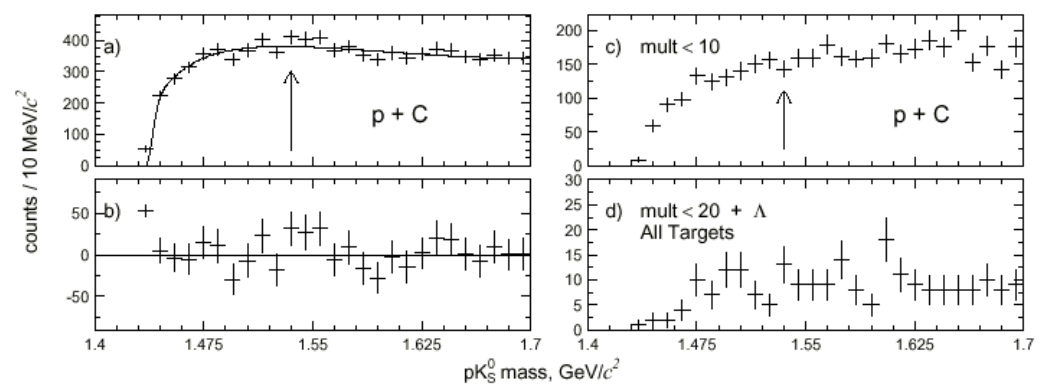

Figure 20: Invariant mass of a $p K^{0}$ pair from the HERA-B experiment [15] for a 920 $\mathrm{GeV}$ proton beam incident on a Carbon target. The top left is for all events, and the top right is for a cut on the multiplicity less than 10 .
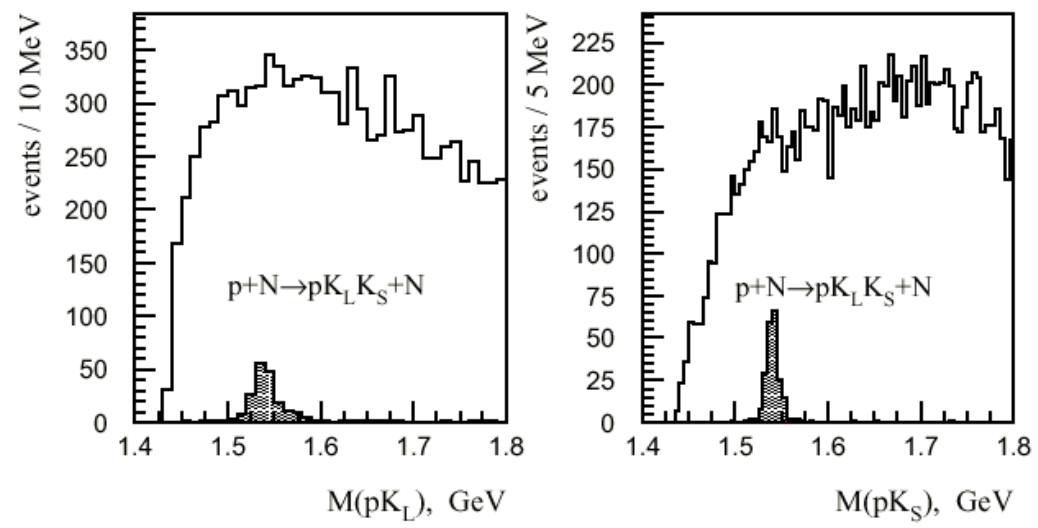

Figure 21: Data from SPHINX [16] for the reaction $p A \rightarrow p K_{s} K_{L} X$ at $70 \mathrm{GeV}$. The invariant mass of both $p K_{L}$ and $p K_{s}$ systems are shown along with a simulation of the $\Theta^{+}$peak position (shaded).

$p K_{L}$ and $p K_{s}$ combinations, along with a simulation of the $\Theta^{+}$peak resolution (shaded). There is no statistically significant structures, same as HERA-B. The SPHINX experiment measured, at the same time, the $p C \rightarrow n K^{+} K_{s} X$ where the neutron was detected in their calorimeter. Again, there is no structure to the $n K^{+}$mass spectrum.

The HyperCP experiment at Fermilab is designed to measure CP violation in cascade $(\Xi)$ and anti-cascade decays. A subset of their data was extracted for a $\Theta^{+}$search from reactions on their tungsten collimator from a beam of mainly protons and $\pi^{+}$with momenta in the range of $100-250 \mathrm{GeV} / \mathrm{c}$. The experiment did not have particle identification. The $K_{s}^{0}$ was reconstructed using the twopion decay angles (only one pion went into their calorimeter). They assume that any charged track with more than $50 \%$ of the momentum is the proton. Clearly, this is not an ideal setup for a pentaquark search. (The authors argue that the broad momentum spread in the beam is an advantage, but the reader can make their own decision.)

The HyperCP results are shown in Fig. 22 along with a simulation showing their expected resolution for a $\Theta^{+}$in their detector. The limit given in Table 2 


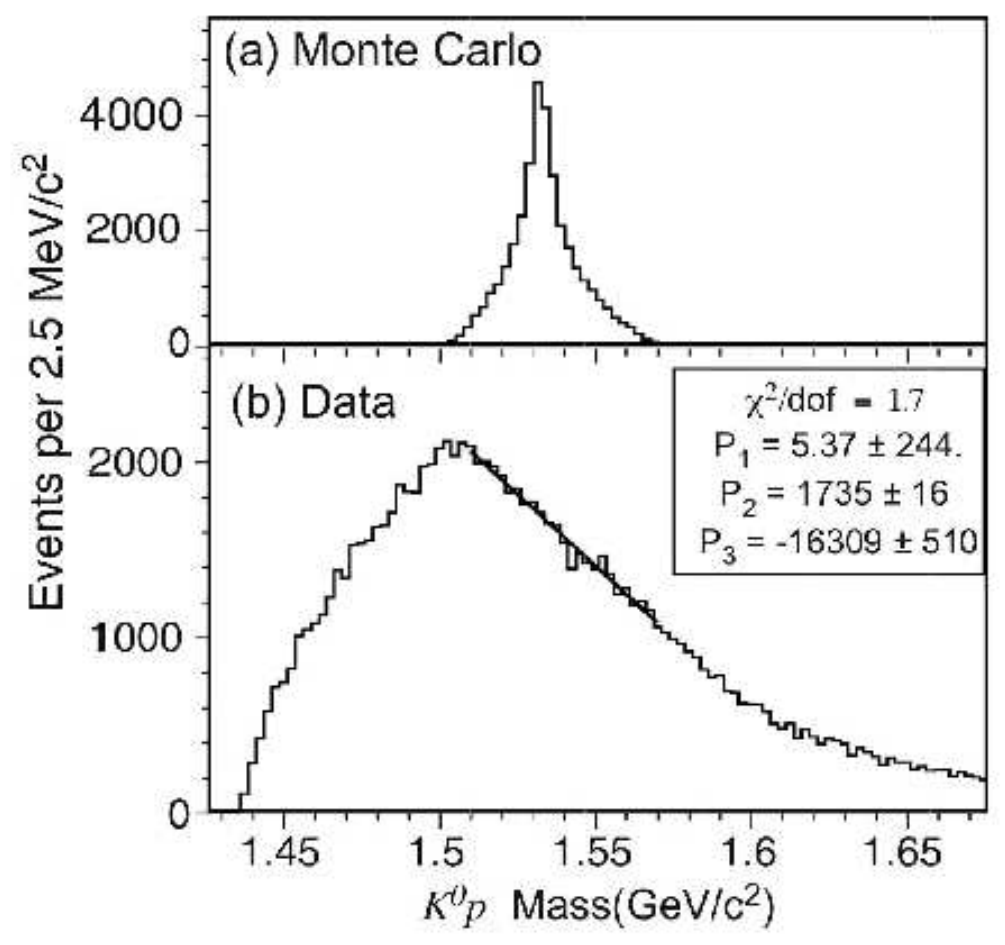

Figure 22: Data from HyperCP [17] for a mixed proton and pion secondary beam scattering from a tungsten collimator. The invariant mass of a reconstructed $K_{s}^{0}$ and proton candidates are shown in the lower plot, along with a simulation of the expected shape of a $\Theta^{+}$peak on top.

assumes that the $\Theta^{+}$is centered at $1.53 \mathrm{GeV}$ only (no limit was given for a $\Theta^{+}$ at $1540 \mathrm{MeV}$, or at $1555 \mathrm{MeV}$ as in the CLAS proton result). Note that it is possible that their spectrum could be contaminated with mis-identified protons that are really pions or kaons (since there is no particle identification). The above concerns suggest that one should carefully evaluate the usefulness of the HyperCP result.

The CDF detector is well-known as a premier tool of high-energy physics. It was good particle identification and excellent resolution. They measured the $p \bar{p} \rightarrow p K^{0} X$ at a center-of-mass energy of nearly $2 \mathrm{TeV}$. This results in a large multiplicity, but the detector has the capability of resolving clean samples of protons and $K_{s}^{0}$ 's. The invariant mass of the $p K^{0}$ system is shown in Fig. 23] with a bin size of $2 \mathrm{MeV}$. A fit to the background that excludes the region from 1.51-1.56 GeV is shown by the solid line, and a search for a peak anywhere in this region was done. Obviously, the result is null whereas they get several thousand counts for the $\Lambda(1520)$ in their $p K^{-}$spectrum. Note that the minibias data used here were heavily prescaled, resulting in lower statistics than other physics triggers. Their paper [18] does not give the multiplicity of particles in each event, and it would be interesting to know more details about the combinatoric backgrounds.

The FOCUS experiment [19] used a photon beam from brehmsstrahlung of $300 \mathrm{GeV}$ electrons and positrons. The photons hit nuclei in a $\mathrm{BeO}$ target, 


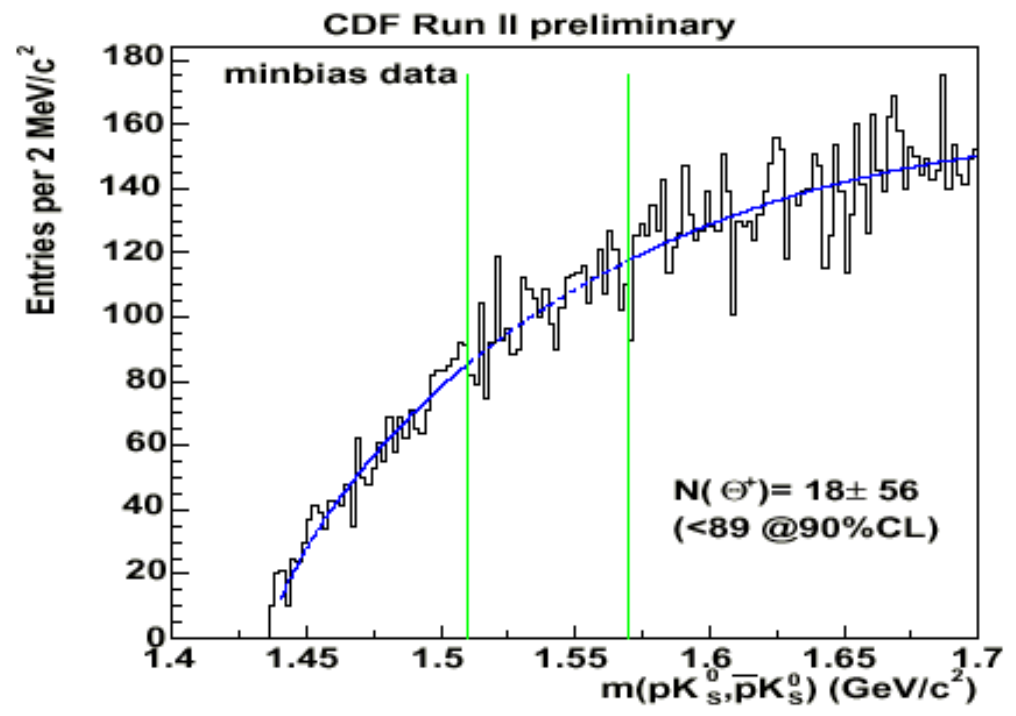

Figure 23: Data from CDF [18] for the reaction $p \bar{p} \rightarrow p K^{0} X$ for their minibias trigger. The shape of the background was fit to the region outside the horizontal lines, and an upper limit on possible $\Theta^{+}$production was estimated for the range inside the horizontal lines.

and charged particles are tracked using a silicon vertex detector followed by momentum analysis in dipole magnets. Particle identification is clean, done by three Cerenkov detectors and two EM calorimeters. Their results are shown in Fig. 24 for the invariant mass of the $p K_{s}^{0}$ system. Few details are given about this analysis, such as estimates for the shape of this spectrum from MC simulations or why they don't see any $\Sigma^{*}$ resonances in this spectrum, but a longer paper with details is expected in the near future.

The Belle experiment 20 took a different approach. They used secondary scattering of mesons (from $e^{+} e^{-}$collisions) in their silicon vertex detector to produce known $Y^{*}$ resonances. If the $\Theta^{+}$exists, it could be produced with a $K^{+}$ beam of the right energy. Their results are shown in Fig. 25. Unfortunately, the hadrons incident on the silicon target have unknown identity and unknown energy. Only a small fraction of these data could result in production of the $\Theta^{+}$ and detected by its decay into the $p K^{0}$ channel. With the high resolution of Belle, even a small signal (with a narrow width) might be visible, but none was seen. Again, we need better calculations of the expected number of counts (based on Belle's spectrum of hadrons incident on silicon) before we can interpret their null result.

\subsection{Discussion of null results}

Based on the high-energy data, one might conclude that no $\Theta^{+}$exists and also that no $\Sigma^{*}$ resonances exist. But what is the production mechanism of the $\Sigma^{*}$ resonances? Similarly, what is the production mechanism of the possible $\Theta^{+}$ resonance? Theoretical calculations are needed in order to understand the true significance of the null results. 


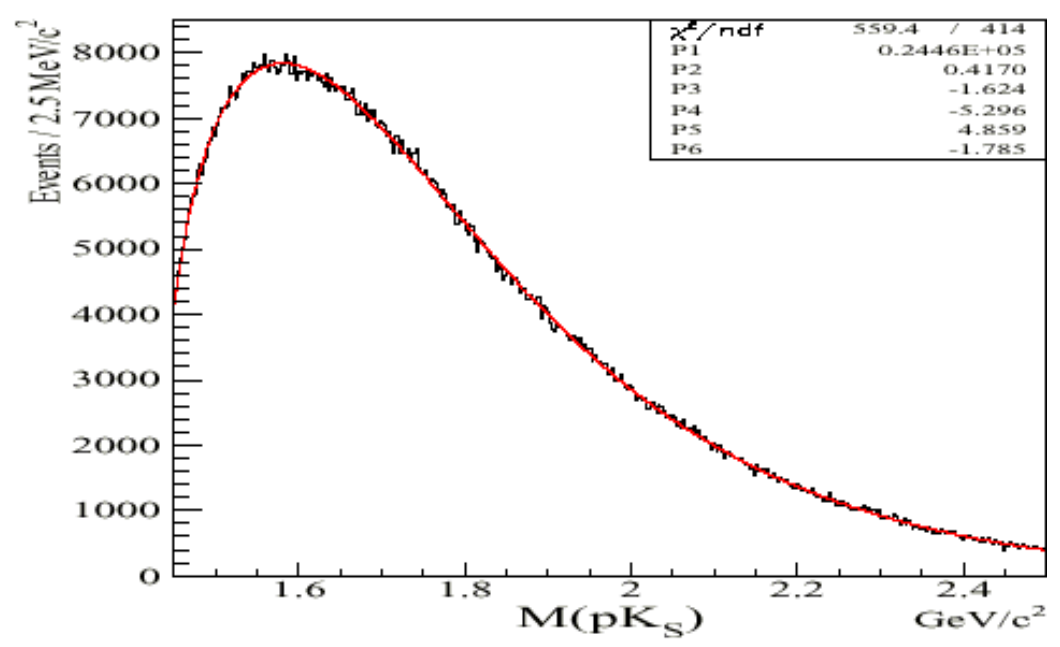

Figure 24: Data from the FOCUS experiment for the reaction $\gamma B e O \rightarrow p K_{s}^{0} X$ from a $300 \mathrm{GeV}$ brehmsstrahlung beam. No structures are seen here, but other channels such as $\Sigma^{*} \rightarrow \Lambda \pi$ have robust peaks.

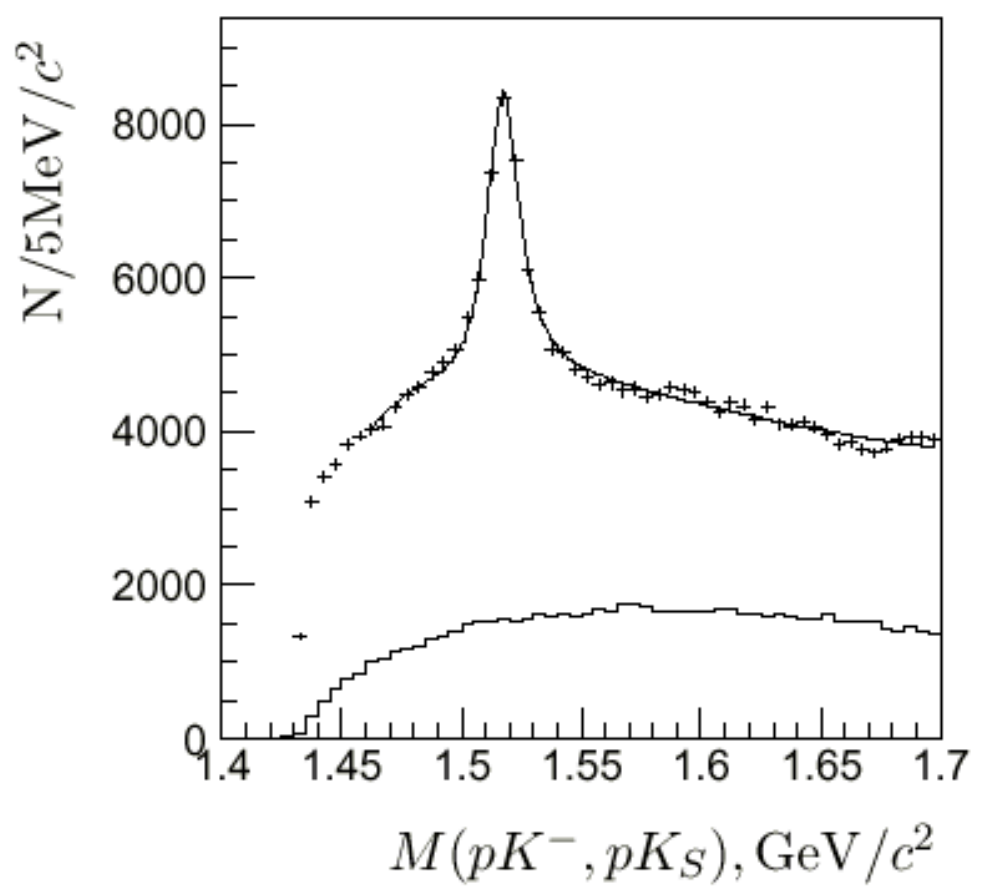

Figure 25: Data from Belle 20] for a beam of secondary particles (mostly protons and pions) from the interaction vertex scattered from their silicon vertex detector. The invariant mass of the $p K^{-}$system is shown with the $\Lambda(1520)$ peak above the spectrum of the $p K_{S}^{0}$ system where no structure is seen. 
The production mechanism of the $\Theta^{+}$(if it exists) or even the $\Lambda^{*}$ and $\Sigma^{*}$ resonances from fragmentation processes is not well known. However, a first step in this direction was taken by Titov et al. [70, using quark constituent counting rules to estimate the ratio of $\Theta^{+}$to $\Lambda(1520)$ production in fragmentation reactions. Fragmentation functions are well established [7] and have been used for years to describe the distribution of hadrons from high-energy collisions, based on the number of constituent partons in the projectile and target. Using this model, Titov shows that production of the $\Theta^{+}$is suppressed relative to the $\Lambda(1520)$ resonance by about 3 orders of magnitude for experiments such as HERA-B. Of course, the simple model used for this estimate may not be a good approximation for all kinematics, but it is consistent with the null experimental results at high energies.

Finally, the facts should be clearly stated when drawing conclusions from both positive and null evidence. The kinematics in the null experiments are different from those in the experiments reporting positive evidence. In other words, the null results do not prove that the positive results are wrong. There may be some interesting physics to be learned, assuming all experiments are correct, as to why exclusive measurements at medium energy show a possible $\Theta^{+}$peak whereas its production may be suppressed in high-energy inclusive measurements. In any case, the onus is on the medium-energy experiments to make a better case for the possible existence of the $\Theta^{+}$.

\section{The Problem of the Width}

Perhaps the most disturbing fact of the $\Theta^{+}$evidence is that its width appears to be very narrow. Direct evidence [2, 7, 8, limits the width to be less than about $10 \mathrm{MeV}$. Indirect evidence, based on analysis of $\mathrm{KN}$ scattering data 45, 48, 46, 49, 50, estimates the width at a few MeV or less. Such a narrow width for a resonance $100 \mathrm{MeV}$ above its strong decay threshold would be unprecedented.

Coupled with the narrow width problem is the question of parity. The spin of the lowest-lying $\Theta^{+}$is expected to be $J=1 / 2$ with either negative (S-wave) or positive (P-wave) parity. A narrow width from an $\mathrm{S}$-wave resonance makes no sense [72] whereas a $\mathrm{P}$-wave would allow a centrifugal barrier so that a narrow width at least possible [72, 73. It seems logical that if the $\Theta^{+}$width is narrow, its parity must be positive. This idea was beautifully presented by Hosaka [7].

What do lattice QCD calculations say about the parity? Several lattice results are known [33] and except for one result [75], only the negative parity projection gives a signal consistent with the $\Theta^{+}$state. So we have an apparent contradiction between the parity deduced from quark models (above) and the parity deduced from (most) lattice calculations. One obvious resolution to this dilemma is to conclude that the $\Theta^{+}$does not exist. In fact, more recent lattice studies with more CPU power have concluded that there is no mass eigenstate that is separable from the low-lying scattering states [76, 77]. However, we must realize that the lattice calculations for exotic baryon resonances should be regarded as exploratory [33. Extrapolating to the chiral limit from the 
heavy quarks used in lattice calculations must be done properly [75, 76] and furthermore, all lattice calculations are done in the quenched approximation. Hence we should be wary of parity statements based on current lattice results.

Even if the $\Theta^{+}$exists with $J^{P}=\frac{1}{2}^{+}$, a width as narrow as $1 \mathrm{MeV}$ is theoretically difficult to understand [78. However, several new theoretical ideas show that such a narrow width is consistent with theory. Ellis, Karliner and Praszalowicz [79] have shown that, when higher-order terms from mixing of the low-lying baryon multiplets due to $\mathrm{SU}(3)$ symmetry breaking are included in the chiral soliton model, the $\Theta^{+}$width could accidentally be very small. Separately, using a two-state model, Karliner and Lipkin showed [80] that the mass eigenstates of two pentaquarks (e.g., mixtures of the Jaffe-Wilczek model and the diquark-triquark model) can mix, resulting in one coupling strongly to KN decay (with a wide width) and one decoupling (with a narrow width). In a different approach, using the QCD string model, Suganuma et al. showed 81] that the pentaquark does not just "fall apart" as predicted by the quark model, but must overcome a sizable potential barrier to decay into a $\mathrm{KN}$ final state. This results in a very narrow width for the $\Theta^{+}$in their model. In all, it is interesting that a narrow width of $1 \mathrm{MeV}$ can be accommodated within the quark model, the chiral soliton model and the QCD string model.

Of course, the $\Theta^{+}$does not necessarily have spin $J=1 / 2$. It is possible that it could have $J=3 / 2$, and this was investigated on the lattice by the Adelaide lattice group [82]. Interestingly, they find attraction for $J^{P}=\frac{3}{2}^{+}$but not in the negative parity state. Also, they do not see any attraction for $J=1 / 2$ in either parity. This is an interesting development, and could be tested experimentally, should the $\Theta^{+}$turn out to be real (with a narrow width).

Clearly, experimental information is needed before one can test the various ideas about the $\Theta^{+}$width. Proposals at KEK [55] and Jefferson Lab [83] for high resolution spectrometer experiments have been approved and will likely run in 2005. Other facilities already mentioned (CLAS, ZEUS and COSY-TOF) will gather more statistics, which should enable a better determination of the $\Theta^{+}$width (if it exists). In addition to width measurements, we need to know the $\Theta^{+}$parity. This will likely be done at COSY-TOF using polarized target and polarized beam, which has a clear theoretical interpretation as shown by Hanhart 84]. If the $\Theta^{+}$exists, then we have the experimental tools to learn about its width and parity.

\section{Summary}

After a long dormant period, there is again experimental action in the search for pentaquarks. Currently, there is a lot of interest, both experimental and theoretical, in the subject. Are the peaks near $1535 \mathrm{MeV}$ real or not? If real, then we will learn a lot about QCD. In particular, are correlations between quarks important in nonperturbative solutions of QCD?

The $K^{+} N$ data from the 1960's and 1970's does not show resonance structure near threshold, and this casts some doubt on the existence of the $\Theta^{+}$pentaquark, unless it is has a narrow width of a few $\mathrm{MeV}$ or less. If it is narrow, then this makes the $\Theta^{+}$(if it exists) difficult to understand in terms of current 
theoretical models. This theoretical bias, coupled with the null results from high energy experiments, makes it difficult to believe the $\Theta^{+}$is real. Hence, the ball is in the experimental court. If the $\Theta^{+}$can be shown to exist, then the nay-sayers will be forgotten, and the theorists will find a way to explain its narrow width.

Hadronic physics, and non-perturbative QCD in general, may hold surprises for us. We should seek ways to explore the edges of this terrain, and searching for multiquark states is one way to do this. Regardless of the outcome, the search is worth the effort.

\section{Acknowledgements}

I am indebted to many colleagues, both experimental and theoretical, with whom I have had discussions on this topic. Special thanks go to Takashi Nakano, leader of the LEPS collaboration, and Stepan Stepanyan, co-spokesman of the CLAS deuterium experiment, whom are my close experimental partners. 


\section{References}

[1] T. Nakano et al., (LEPS), Phys. Rev. Lett. 91:012002 (2003); hepex/0301020.

[2] V.V. Barmin et al., (DIANA), Phys. Atom. Nuclei 66, 1715 (2003); hepex/0304040.

[3] S. Stepanyan et al., (CLAS), Phys. Rev. Lett. 91:25001 (2003); hepex/0307018.

[4] J. Barth et al., (SAPHIR), Phys. Lett. B572, 127 (2003); hep-ex/0307083.

[5] A.E. Asratyan, A.G. Dolgolkenko and M.A. Kubantsev, Phys. Atom. Nucl. 67, 682 (2004); hep-ex/0309042.

[6] V. Kubarovsky et al., (CLAS), Phys. Rev. Lett. 92:032001 (2004); hepex/0311046.

[7] A. Airapetian et al., (HERMES), Phys. Lett. B585 (2004) 213; hep$\mathrm{ex} / 0312044$.

[8] The ZEUS collaboration, Phys. Lett. B591 (2004) 7; hep-ex/0403051.

[9] M. Abdel-Barv, et al., (COSY-TOF), Phys. Lett. B595, 127 (2004); hepex/0403011.

[10] A. Aleev et al., (SVD), submitted to Yad. Fiz.; hep-ex/0401024.

[11] J.Z. Bai et al., (BES), Phys. Rev. D bf 70:012004 (2004); hep-ex/0402012.

[12] The BaBar Collaboration, hep-ex/0408064.

[13] The Belle Collaboration, hep-ex/0409010.

[14] S.R. Armstrong, hep-ex/0410080; S. Schael et al., (ALEPH), Phys. Lett. B 599, 1 (2004).

[15] I. Abt et al., (HERA-B), Phys. Rev. Lett. 93:212003 (2003); hepex/0408048.

[16] Yu.M. Antipov et al., (SPHINX), Eur. Phys. J. A21, 455 (2004); hepex/0407026.

[17] M.J. Longo et al., (HyperCP), Phys. Rev. D 70:111101 (2004); hepex/0410027.

[18] D.O. Litvintsev (CDF), hep-ex/0410024.

[19] K. Stenson et al., (FOCUS), hep-ex/0412021.

[20] R. Mizuk et al., (Belle), hep-ex/0411005.

[21] C. Pinkerton et al., (PHENIX), J. Phys. G 30:S1201 (2004); nuclex/0404001.

[22] C. Alt et al., (NA49), Phys. Rev. Lett. 92, 042003 (2004); hep-ex/0310014.

[23] H.G. Fischer and S. Wenig, Eur. Phys. J. C37, 133 (2004); hepex/0401014.

[24] M.I. Adamovich et al., (WA89), Phys. Rev. c 70:022201 (2004); hepex/0405042. 
[25] A. Aktas et al., Phys. Lett. B 588, 17 (2004); hep-ex/0403017.

[26] S. Chekanov et al., (ZEUS), Eur. Phys. J. C38, 29 (2004); hepex/0409033.

[27] Website http://www.rcnp.osaka-u.ac.jp/h̆yodo/research/Thetapub.html

[28] R.L. Jaffe, Phys. Rev. D 15, 267 (1977); preprint SLAC-PUB-1774.

[29] D. Strottman, Phys. Rev. D 20, 748 (1979).

[30] H. J. Lipkin, Nucl. Phys. A 625, 207 (1997).

[31] D. Diakonov, V. Petrov and M. Polyakov, Z. Phys. A 359, 305 (1997).

[32] Colin Morningstar, AIP Conf. Proc. 698 (New York, 2003); nuclth/0308026.

[33] S. Sasaki, hep-lat/0410016.

[34] K. Goeke, H.-C. Kim, M. Praszalowicz, G.-S. Yang, hep-ph/0411195; to be published in Prog. Nucl. Part. Phys.

[35] V.D. Burkert and T.S.H. Lee, nucl-ex/0407020; submitted to Int. J. Mod. Phys. E.

[36] R.L. Workman et al., nucl-th/0404061 and nucl-th/0410110.

[37] J.S. Hyslop et al., Phys. Rev. D 46, 961 (1992).

[38] R.G. Glasser et al., Phys. Rev. D15, 1200 (1977).

[39] T. Bowen et al., Phys. Rev. D 2, 2599 (1970).

[40] T. Bowen et al., Phys. Rev. D 7, 22 (1973).

[41] V.J. Stenger et al., Phys. Rev. 134, B1111 (1964); W. Slater et al., Phys. Rev. Lett. 7, 378 (1961).

[42] A.K. Ray et al., Phys. Rev. 183, 1183 (1969).

[43] T. Barnes and E.S. Swanson, Phys. Rev. C 49, 1166 (1994).

[44] C.J.S. Damerell et al., Nucl. Phys. B94, 374 (1975).

[45] S. Nussinov, hep-ph/0307357.

[46] R.A. Arndt et al., Phys. Rev. C68, 042201 (2003); Erratum C69, 019901 (2004).

[47] J. Haidenbauer and G. Krein, Phys. Rev. C68, 052201 (2003).

[48] R.N. Cahn and G.H. Trilling, Phys. Rev. D69, 011501 (2004).

[49] A. Sibirtsev et al., Phys. Lett. B599, 230 (2004).

[50] W.R. Gibbs, Phys. Rev. C70, 045208 (2004).

[51] R.L. Cool et al., Phys. Rev. D 1, 1887 (1970).

[52] D.V. Bugg et al., Phy. Rev. 168, 1466 (1968).

[53] J. Tyson et al., Phys. Rev. Lett. 19, 255 (1967).

[54] A. Berthon et al., Nucl. Phys. B63, 54 (1973).

[55] K. Imai et al., experiment E559 at the KEK facility in Japan. 
[56] T. Nakano and K. Hicks, Mod. Phys. Lett. A19, 645 (2004).

[57] J. Pochodzalla, hep-ex/0406077.

[58] A. Dzierba et al., Phys. Rev. D69 (2004) 051901.

[59] K. Hicks et al., submitted to Phys. Rev. D; hep-ph/0411265.

[60] K. Hicks, Hadron 2003 Conference, AIP Conference Proceedings No. 717, (New York, 2004), p. 400.

[61] Michael Ostrick, Pentaquark 2003 Workshop, Thomas Jefferson National Accelerator Facility, November 2003.

[62] S. Nam, A. Hosaka, H.-C. Kim, hep-ph/0403009.

[63] W. Liu and C.M. Ko, Phys. Rev. C 68:045203 (2003); nucl-th/0308034. See also Nucl. Phys. A741, 215 (2004); nucl/th/0309023.

[64] Y. Oh, H. Kim and S.H. Lee, Phys. Rev. D 69:014009 (2004), hep$\mathrm{ph} / 0310019$.

[65] W. Lorenzon (HERMES Collaobration), hep-ex/0411027.

[66] S. Chekanov, hep-ph/0502098.

[67] W. Eyrich, Exotic Hadrons Workshop, Trento (Feb. 2005).

[68] Ya. I. Azimov and I.I. Strakovsky, Phys. Rev. C 70:035210 (2004).

[69] Particle Data Group, Review of Particle Properties, Phys. Lett. B 592 (2004).

[70] A.I. Titov, A. Hosaka, S. Date and Y. Ohashi, Phys. Rev. C 70:042202 (2004).

[71] F. Close, Proceedings of the Hadron 2003 Conference, AIP Conf. Proc. 717 (2004), p. 919; hep-ph/0311258.

[72] R. Jaffe and F. Wilczek, Phys. Rev. Lett. 91:232003 (2003).

[73] M. Karliner and H.J. Lipkin, Phys. Lett. B575 (2003) 249; hep$\mathrm{ph} / 0307243$.

[74] S.-I. Nam, A. Hosaka, and H.-C. Kim, hep-ph/0501135.

[75] T.-W. Chiu and T.-H. Hsieh, hep-ph/0403020.

[76] N. Mathur et al., Phys. Rev. D 70:074508 (2004); hep-ph/0406196.

[77] F. Csikor, Z. Fodor, S.D. Kratz, T.G. Kovacs and B.C. Toth, heplat/0503012.

[78] M. Praszalowicz, hep-ph/0410241.

[79] J. Ellis, M. Karliner and M. Praszalowicz, JHEP 0405, 2 (2004); hep$\mathrm{ph} / 0401127$.

[80] M. Karliner and H. Lipkin, Phys. Lett. B586, 303 (2004); hep-ph/0401072.

[81] H. Suganuma, H. Ichie, F. Okiharu, T. Takahashi; hep-ph/0412271.

[82] B.G. Lasscock et al., hep-lat/0504015.

[83] B. Wojtsekhowski and G. Cates, proposal P05-009.

[84] C. Hanhart, J. Haidenbauer, K. Nakayama, U.-G. Meissner, hep$\mathrm{ph} / 0407107$. 